\title{
Quark masses in Higgs production with a jet veto
}

\author{
Andrea Banfi, ${ }^{a}$ Pier Francesco Monni ${ }^{b, c, d}$ and Giulia Zanderighi ${ }^{c}$ \\ ${ }^{a}$ Department of Physics and Astronomy, University of Sussex, \\ Sussex House, Brighton, BN1 9RH, U.K. \\ ${ }^{b}$ Institut für Theoretische Physik, Universität Zürich, \\ Winterthurerstrasse 190, CH-8057 Zürich, Switzerland \\ ${ }^{c}$ Rudolf Peierls Centre for Thoeretical Physics, University of Oxford, \\ 1 Keble Road, Oxford OX1 3NP, U.K. \\ ${ }^{d}$ Institute for Particle Physics Phenomenology, University of Durham, \\ South Road, Durham DH1 3LE, U.K. \\ E-mail: a.banfi@sussex.ac.uk, pfmonni@physik.uzh.ch, \\ g.zanderighi1@physics.ox.ac.uk
}

ABSTRACT: We study the impact of finite mass effects due to top and bottom loops in the jet-veto distribution for Higgs production. We discuss the appearance of non-factorizing logarithms in the region $p_{\text {t,veto }} \gtrsim m_{b}$. We study their numerical impact and argue that these terms can be treated as a finite remainder. We therefore detail our prescription for resumming the jet-vetoed cross section and for assessing its uncertainty in the presence of finite mass effects. Resummation for the jet-veto, including mass effects, has been implemented in the public code JetVHeto.

Keywords: Jets, Hadronic Colliders

ARXIV EPRINT: 1308.4634 


\section{Contents}

1 Introduction 1

2 Kinematics and cross sections 4

3 Factorization of soft and collinear singularities $\quad 6$

$\begin{array}{lll}3.1 \text { Soft limit } & 6\end{array}$

3.2 Collinear limit 8

4 Factorization and resummation formula $\quad 9$

$\begin{array}{llr}4.1 & \text { Resummation formula } & 9\end{array}$

$\begin{array}{ll}4.2 \text { Treatment of non-factorizing terms } & 10\end{array}$

5 Phenomenology 14

$\begin{array}{llr}6 & \text { Conclusions } & 19\end{array}$

$\begin{array}{ll}\text { A Real emission matrix elements } & 21\end{array}$

$\begin{array}{ll}\text { B Matching schemes } & 23\end{array}$

$\begin{array}{ll}\text { C Mod-(a) matching scheme } & 24\end{array}$

D Comparison to the Higgs transverse momentum 26

\section{Introduction}

A new particle with mass $m_{H} \sim 125 \mathrm{GeV}$ was discovered last year at the LHC $[1,2]$. After just one year from its discovery, precision measurements of the characteristics of this particle have already been carried out: its mass is known with better than $1 \mathrm{GeV}$ precision, and all properties studied so far are compatible with the particle being the Higgs boson of the Standard Model (SM). This marks a remarkable success of the experiment. However, contrary to expectations, no evidence for New Physics has been found yet at the LHC. Since New Physics is elusive, it is natural to focus on the newly discovered particle and to perform as accurate as possible phenomenological studies on it. In order to fully establish the nature of the new particle as the SM Higgs boson, even more precise measurements of its properties, in particular its couplings, are essential. Establishing a significant departure from the simple SM-like pattern could rule out the possibility that the new particle is the plain SM Higgs boson, and could be a first manifestation of New Physics.

In fact, the new particle lies in a sweet spot where many decay modes have an appreciable branching fraction, leading to a very rich phenomenology. Second only to the 
branching to $b \bar{b}$, which however is very difficult experimentally because of the large $g g \rightarrow b \bar{b}$ background, is the branching to $W W$. In this channel, a dominant, reducible background comes from top-pair production, where each top decays to a $W$-boson and a $b$-quark that generally gives rise to a jet. On the other hand, most Higgs-boson events involve either none or one jet, since higher multiplicity events are suppressed by powers of the strong coupling constant, and hence more rare. It is natural then to impose a veto on additional jets so as to considerably reduce the dominant top background, while reducing only modestly the signal. Still, it is then essential to understand how much these tight kinematical cuts reduce the signal cross-section, and what are the uncertainties associated to efficiencies and cross-sections in the presence of jet-vetoes.

Perturbative predictions involve, for each power of the coupling constant, double logarithms of the jet-veto scale $p_{\mathrm{t} \text {,veto }}$ and of the scale of the hard scattering $m_{H}$. ATLAS and CMS use jet-veto scales of the order of $25-30 \mathrm{GeV}$, so that in order to make accurate predictions it becomes important to account for these logarithms to all orders in perturbation theory. In recent years, a lot of progress has been made both in understanding and computing resummed predictions for the jet-veto cross section. In ref. [3], it was pointed out that the jet-veto is within the scope of the resummation program CAESAR [4] and a next-to-leading logarithmic (NLL) resummation matched to next-to-next-to-leading order (NNLO) results was presented. Subsequently, a full NNLL+NNLO resummation was computed in $[5,6]$ and very recently also in $[7,8]$, including partial $\mathrm{N}^{3} \mathrm{LL}$ terms. However, a full $\mathrm{N}^{3} \mathrm{LL}$ resummation requires still some work, among which the exact calculation of the four-loop cusp anomalous dimension (rather than its Padé approximation) and the exact coefficient of the relative $\alpha_{s}^{3} L$ term.

Residual uncertainties for jet-veto efficiencies for a $25-30 \mathrm{GeV}$ jet-veto are of the order of $\pm 10 \%$. These uncertainties propagate into the jet-veto cross-section, giving rise to uncertainties of the order of $\pm 11-12 \%$ (for $8 \mathrm{TeV}$ collisions, using the anti- $k_{t}$ algorithm [9] with $R=0.5$ with $\left.p_{\mathrm{t}, \text { veto }}=25-30 \mathrm{GeV}\right)$. This should be compared with a $\pm 15-20 \%$ uncertainty in fixed-order NNLO predictions. While the resummation is successful in reducing the uncertainties in this region, one needs to bear in mind that all these resummed predictions use the large- $m_{t}$ effective theory, where the Higgs couples directly to gluons via an effective vertex, and bottom quark loops are completely neglected. The above uncertainties do not account for the error associated with the large- $m_{t}$ approximation. Given the high accuracy reached by these resummed predictions, it becomes then important to assess the impact of finite-mass effects on jet-vetoed cross-sections and the associated theoretical error.

The impact of finite mass corrections in Higgs cross-sections or Higgs transverse momentum distributions has been studied already in quite some detail at fixed order [10-16]. Exact finite-mass effects for Higgs production accompanied by one jet have been also implemented at LO in the code MCFM [17]. In ref. [18], HPRO, a NLO Monte-Carlo for Higgs production via gluon fusion with finite heavy-quark masses was presented. In [19] the program SusHi is presented, that contains NLO QCD contributions from the third family of quarks and squarks, NNLO corrections due to top-quarks, approximate NNLO corrections due to stops, and electro-weak effects. The contribution of initial-state bottom quarks has been computed at NNLO in refs. [20-23] and it was found to be small. Furthermore, 
finite quark-mass effects are included in Monte Carlo generators like Herwig [24, 25] and Pythia [26] and have recently been implemented also in the NLO Monte Carlo event generators POWHEG [27] and MC@NLO [28].

In general, for inclusive cross-sections one finds that bottom-mass effects are important and opposite in sign to the top-mass effects. In fact, while the pure top contribution gives rise at NLO to about a $6 \%$ correction to the inclusive cross-section, once both top and bottom effects are included the correction with respect to the heavy top limit is about $-2 \%$. Both top and bottom mass effects distort the Higgs transverse momentum spectrum considerably, and therefore cannot be included as a $K$-factor. In particular at large transverse momenta corrections become large and negative. Furthermore, while POWHEG and MC@NLO agree well with each other when only exact top-quark mass effects are accounted for, when also $b$-mass effects are included, the two approaches give considerably different predictions for $p_{t, H} \lesssim 50 \mathrm{GeV}$, i.e. in the region where resummation effects are important. This is not surprising since in the POWHEG approach higher order terms in the Sudakov are exponentiated, while in MC@NLO they are not. If these higher order effects are important, the two methods will give numerically different answers.

An analytic resummation for the Higgs transverse momentum distribution including mass effects have been considered recently at NLL+NLO in ref. [29]. Finite top- and bottom-quark masses up to $\mathcal{O}\left(\alpha_{s}^{3}\right)$ have also been included in the NNLL+NNLO transverse momentum spectrum of the Higgs boson [30]. This reference also discusses difficulties arising in the case of bottom quarks, i.e. when the heavy-quark mass is much smaller than the Higgs mass. In fact, in this case the two-scale problem (the two scales being $p_{t, H}$ and $m_{H}$ ) that is treated using standard resummation techniques becomes a three-scale problem (the third scale being the bottom mass $m_{b}$ ). The approach of ref. [30] is based on the observation that collinear factorization is valid only when $p_{t, H} \lesssim m_{b}$. Hence, the authors of ref. [30] introduce a second resummation scale which controls the impact of the resummation of bottom quark contributions. They set this scale to $m_{b}$ hence limiting the resummation only to the region $p_{t, H} \lesssim m_{b}$.

In this paper, we study the "factorization breaking terms" which appear for $p_{t} \gtrsim m_{b}$ further and argue that, in our approach, it is not necessary to switch off the resummation in this region. We also show that these terms can be safely treated as any other nonfactorizing finite remainder in our resummation formalism.

The paper is organized as follows. In section 2 we describe the kinematics of the problem and introduce the jet-veto observable. In section 3 we discuss the (non)-factorizing terms appearing when considering soft and collinear limits of the Higgs plus one parton matrix elements. In section 4 we assess the numerical impact of these terms, discuss in detail the prescription we use to treat them and present our resummation formula including finite-mass effects. In particular, we discuss whether it is appropriate, and what is the impact of changing the resummation scale for the bottom induced contribution and we add an additional logR-type matching scheme, whose purpose is to explore the impact of exponentiating, or not, non-factorizing terms whose higher order structure is unknown. In section 5 we present phenomenological results for the LHC with realistic cuts as applied by ATLAS and CMS. We pay particular attention to quantifying the impact 
of top and bottom mass effects by comparing with the large- $m_{t}$ approximation and to assessing theory uncertainties. We conclude in section 6. Appendix A collects results for real emission matrix element squared, appendix B collects our standard matching formulae, and appendix $\mathrm{C}$ presents an additional matching scheme.

\section{Kinematics and cross sections}

We consider the production of a Higgs boson accompanied by an arbitrary number of extra QCD partons

$$
p_{1} p_{2} \rightarrow p_{H} p_{3}, \ldots, p_{n} .
$$

A jet-veto condition is imposed by clustering the final state partons into jets using an infrared and collinear safe jet-algorithm, and requiring that each event has no jets with transverse momentum above $p_{\mathrm{t}, \text { veto }}$. The jet-veto efficiency $\epsilon\left(p_{\mathrm{t}, \text { veto }}\right)$ is defined as the fraction of events that pass the jet-veto condition:

$$
\epsilon\left(p_{\mathrm{t}, \mathrm{veto}}\right)=\frac{\Sigma\left(p_{\mathrm{t}, \mathrm{veto}}\right)}{\sigma_{\mathrm{tot}}} .
$$

Here $\Sigma\left(p_{\text {t,veto }}\right)$ is the so-called zero-jet cross section, defined as

$$
\Sigma\left(p_{\mathrm{t}, \mathrm{veto}}\right)=\sum_{n} \int d \Phi_{n} \frac{d \sigma_{n}}{d \Phi_{n}} \Theta\left(p_{\mathrm{t}, \mathrm{veto}}-p_{\mathrm{t}, \mathrm{jet}}\right)
$$

where $p_{\mathrm{t} \text {,jet }}$ is the transverse momentum of the hardest jet, $d \sigma_{n}$ is the cross section for producing a Higgs and $n-2$ extra partons, and $d \Phi_{n}$ is the corresponding phase space. Moreover, $\sigma_{\text {tot }}$ is the total Higgs production cross section. Both $\sigma_{\text {tot }}$ and $\Sigma\left(p_{\text {t,veto }}\right)$ can be expanded in powers of $\alpha_{s}$

$$
\begin{aligned}
\Sigma\left(p_{\mathrm{t}, \text { veto }}\right) & =\Sigma_{0}\left(p_{\mathrm{t}, \text { veto }}\right)+\Sigma_{1}\left(p_{\mathrm{t}, \text { veto }}\right)+\Sigma_{2}\left(p_{\mathrm{t}, \text { veto }}\right)+\ldots, \\
\epsilon\left(p_{\mathrm{t}, \mathrm{veto}}\right) & =\epsilon_{0}\left(p_{\mathrm{t}, \text { veto }}\right)+\epsilon_{1}\left(p_{\mathrm{t}, \text { veto }}\right)+\epsilon_{2}\left(p_{\mathrm{t}, \text { veto }}\right)+\ldots, \\
\sigma_{\text {tot }} & =\sigma_{0}+\sigma_{1}+\sigma_{2}+\ldots
\end{aligned}
$$

At the lowest order, $\alpha_{s}^{2}, \Sigma_{0}\left(p_{\text {t,veto }}\right)$ is just the LO cross section $\sigma_{0}$. At higher orders, we obtain the zero-jet cross section from the differential distribution in $p_{\mathrm{t} \text {,jet }}$ as follows

$$
\Sigma_{i}\left(p_{\mathrm{t}, \text { veto }}\right)=\sigma_{i}+\bar{\Sigma}_{i}\left(p_{\mathrm{t}, \text { veto }}\right), \quad \bar{\Sigma}_{i}\left(p_{\mathrm{t}, \text { veto }}\right)=-\int_{p_{\mathrm{t}, \text { veto }}}^{\infty} d p_{t} \frac{d \Sigma_{i}}{d p_{t}} .
$$

Starting from NLO, each $\Sigma_{i}\left(p_{\text {t,veto }}\right)$ contains large logarithmic corrections up to $\alpha_{s}^{2+i} L^{2 i}$, with $L=\ln \left(m_{H} / p_{\text {t,veto }}\right)$. In ref. [6], such logarithmic contributions have been resummed in the context of the CAESAR approach [4] up to NNLL accuracy, i.e. accounting for all logarithmically enhanced terms down to $\alpha_{s}^{n} L^{n-1}$ in $\ln \left(\Sigma / \sigma_{0}\right)$. The basis of the resummation in ref. [6] is the factorization of soft and collinear singularities from the Born matrix element. At tree level, the Higgs is produced via gluon fusion through a top loop. If the top-quark mass $m_{t}$ is much larger than all other scales in the problem, i.e. the large- $m_{t}$ limit, factorization of soft and collinear singularities is valid since soft or collinear partons 
can never resolve the top loop. However, when the transverse momentum of the emitted gluons is larger than the mass of the virtual quarks, new logarithms of the ratio of the two scales appear in the perturbative expansion. Such logarithms are not proportional to the Born cross section, and in the following we will refer to them as non-factorizing terms. In the case of light quarks, i.e. bottom quarks, these logarithmic terms can potentially have an important impact in the region of phenomenological interest. ${ }^{1}$

The appearance of these new logarithms can be observed already at the Born level for the case of Higgs production with no extra partons. Let us first consider a single quark loop, and let $m_{H}$ and $m$ denote the Higgs and quark mass, respectively. The amplitude squared for the process is given by

$$
\left|M_{B}\right|^{2}=\left(N_{c}^{2}-1\right) \frac{y^{2} \alpha_{s}^{2}}{64 \pi^{2}}\left(\left|M_{++}\right|^{2}+\left|M_{--}\right|^{2}\right),
$$

where $y=g_{w} m /\left(2 M_{W}\right)$ is the quark Yukawa coupling, and $M_{++}, M_{--}$are the only two non-vanishing helicity amplitudes, since the helicity of the incoming gluons has to be the same due to angular momentum conservation. Furthermore thanks to parity conservation $M_{++}=M_{--}$.

The amplitude $M_{++}$(taken from ref. [31] eq. (9.34)) is given by

$$
M_{++}=8 m\left[\left(1-\frac{4 m^{2}}{m_{H}^{2}}\right) \frac{1}{2} m_{H}^{2} C\left(m_{H}^{2}\right)-1\right],
$$

where $C\left(m_{H}^{2}\right)$ is the scalar triangle integral in eq. (A.8). If $m^{2} \gg m_{H}^{2}$ we recover the well-known large- $m$ limit,

$$
\left|M_{B}^{\infty}\right|^{2}=\left(N_{c}^{2}-1\right) \frac{G_{F} m_{H}^{4}}{9 \sqrt{2}} \frac{\alpha_{s}^{2}}{\pi^{2}}
$$

If $m^{2} \ll m_{H}^{2}$ the triangle integral reads

$$
m_{H}^{2} C\left(m_{H}^{2}\right)=\frac{1}{2} \ln ^{2}\left(-\frac{m_{H}^{2}}{m^{2}}\right)+\mathcal{O}\left(\frac{m^{2}}{m_{H}^{2}}\right)
$$

which gives

$$
M_{++} \simeq 8 m\left[\frac{1}{2} m_{H}^{2} C\left(m_{H}^{2}\right)-1\right] \simeq 2 m \ln ^{2}\left(-\frac{m_{H}^{2}}{m^{2}}\right) .
$$

The physical origin of the result above can be understood by examining the structure of the quark loop. First of all, the Higgs-quark vertex does not conserve helicity, therefore the amplitude has to be proportional to the quark mass $m$. When the loop momentum probes the kinematical region between the two relevant scales (the quark mass $m$ and the Higgs mass $m_{H}$ ), the loop integral exhibits the usual (soft-collinear) double logarithmic behaviour.

If one extra gluon with transverse momentum $p_{t} \geq m$ is emitted off the fermion line running in the loop, then one expects to observe double logarithms of the type $\ln ^{2}\left(p_{t}^{2} / m^{2}\right)$. For

\footnotetext{
${ }^{1}$ The Yukawa coupling and explicit mass dependence of quark-loops suppress the impact of yet lighter quarks.
} 
the jet-veto cuts applied by ATLAS and CMS, $p_{\mathrm{t} \text {,veto }}=25-30 \mathrm{GeV}, p_{\mathrm{t} \text {,veto }} / m_{b} \sim m_{H} / p_{\mathrm{t} \text {,veto }}$, therefore these logarithms can be as large as the logarithms $\ln \left(m_{H} / p_{\text {t,veto }}\right)$ that we want to resum. Furthermore these logarithms are not proportional to the Born amplitude in eq. (2.10), meaning that soft radiation does not factorize in the regime $m^{2} \ll p_{t}^{2}$. Since this fact questions the basis of our resummation approach, we devote the next section to the calculation of these non-factorizing corrections to lowest order in perturbation theory.

\section{$3 \quad$ Factorization of soft and collinear singularities}

We consider here the amplitude for Higgs production in association with one extra parton

$$
p_{1} p_{2} \rightarrow p_{H} p_{3} \text {. }
$$

At lowest order in perturbation theory this process was computed in [32, 33]. It has three contributing subprocesses, $g g \rightarrow H g, q g \rightarrow H q$, and $g \bar{q} \rightarrow H \bar{q}$. These subprocesses proceed through gluon fusion via a quark loop. The case when the mass of the virtual quark is comparable to or heavier than the Higgs mass is well-known. We wish to investigate the behaviour of the amplitudes in presence of a light quark of mass $m \ll m_{H}$ when the extra parton of transverse momentum $p_{t}$ is either soft or collinear. We are interested in both cases $m \ll p_{t} \ll m_{H}$ and $p_{t} \ll m \ll m_{H}$.

\subsection{Soft limit}

We consider first the case in which the emitted parton $p_{3}$ is soft. As expected, and by direct inspection of the amplitudes in appendix A, only the subprocess $g g \rightarrow H g$ exhibits a soft singularity. It is useful to split the amplitude into different contributions according to the helicity configurations $\lambda_{1}, \lambda_{2}, \lambda_{3}$ of the involved gluons $p_{1}, p_{2}$ and $p_{3}$, with $\lambda_{i}= \pm$. Relations among the various helicity amplitudes $M_{g g \rightarrow H g}^{\lambda_{1} \lambda_{2} \lambda_{3}}$ can be found in appendix A, eqs. (A.12) and (A.13).

Due to angular momentum conservation, in the soft limit, only two of the four independent amplitudes are non-zero, namely $M_{g g \rightarrow H g}^{+++}$and $M_{g g \rightarrow H g}^{++-}$(see eqs. (A.4) and (A.5)). In this limit, the invariants $s, t, u$ in eq. (A.1), and $s_{1}, u_{1}, t_{1}$ in eq. (A.2) reduce to

$$
s \rightarrow m_{H}^{2}, \quad t, u \rightarrow 0, \quad s_{1} \rightarrow 0, \quad t_{1}, u_{1} \rightarrow-m_{H}^{2} .
$$

Furthermore, in the soft limit, we keep only the leading terms proportional to $1 /(u t)=$ $1 /\left(m_{H}^{2} p_{t}^{2}\right)$ and drop all terms proportional to $m^{2}$. This gives

$$
\begin{aligned}
& M_{g g \rightarrow H g}^{+++} \simeq-\frac{64 m y \Delta}{u t}\left\{1-\frac{1}{2} m_{H}^{2} C\left(m_{H}^{2}\right)\right. \\
&\left.+\frac{1}{4}\left[t C(t)+u C(u)-\frac{1}{2}[s t D(s, t)+u s D(u, s)-u t D(u, t)]\right]\right\},
\end{aligned}
$$

and

$$
\begin{aligned}
M_{g g \rightarrow H g}^{++-} \simeq \frac{64 m y \Delta}{u t} & \left\{1-\frac{1}{2} m_{H}^{2} C\left(m_{H}^{2}\right)\right. \\
+ & \left.\frac{1}{4}\left[t C(t)+u C(u)-\frac{1}{2}[s t D(s, t)+u s D(u, s)+u t D(u, t)]\right]\right\},
\end{aligned}
$$


where $\Delta=\sqrt{(s t u) / 8}$. Notice that both amplitudes contain a factorizing term (first line) that is proportional to the Born amplitude eq. (2.7), and an additional term, which we call a non-factorizing correction (second line). If the latter term does not vanish in the limit $p_{t} \rightarrow 0$ then the factorization of soft radiation is violated. Its expression depends on the behaviour of the scalar integrals $C$ and $D$, which is different in the two regimes, $p_{t}^{2} \ll m^{2} \ll m_{H}^{2}$ and $m^{2} \ll p_{t}^{2} \ll m_{H}^{2}$.

1. $p_{t}^{2} \ll m^{2} \ll m_{H}^{2}$. In this limit, the contributions from all $C$ and $D$ integrals vanish (see eqs. (A.8) and (A.10)), except for $m_{H}^{2} C\left(m_{H}^{2}\right)$. As expected, we have factorization of the soft matrix element from the Born amplitude $M_{++}$in eq. (2.7)

$$
M_{g g \rightarrow H g}^{+++}=-M_{g g \rightarrow H g}^{++-} \simeq \frac{8 y \Delta}{u t} M_{++} .
$$

2. $m^{2} \ll p_{t}^{2} \ll m_{H}^{2}$. In this case any triangle integral has the behaviour

$$
q^{2} C\left(q^{2}\right) \simeq \frac{1}{2} \ln ^{2}\left(\frac{m^{2}}{-\left(q^{2}+i \epsilon\right)}\right)
$$

and the box integrals reduce to

$$
\begin{aligned}
s t D(s, t) \simeq & \ln ^{2}\left(-\frac{m^{2}}{t}\right), \quad s u D(s, u) \simeq \ln ^{2}\left(-\frac{m^{2}}{u}\right), \\
u t D(u, t) \simeq & \ln ^{2}\left(-\frac{m^{2}}{t}\right)+\ln ^{2}\left(-\frac{m^{2}}{u}\right)-\ln ^{2}\left(\frac{m^{2}}{m_{H}^{2}}\right)+2 \ln \left(\frac{-t}{m_{H}^{2}}\right) \ln \left(\frac{-u}{m_{H}^{2}}\right) \\
& -2 i \pi\left[\ln \left(\frac{m^{2} m_{H}^{2}}{u t}\right)+2 \ln \left(\frac{m_{H}^{4}}{u t}\right)\right]-\frac{2}{3} \pi^{2} .
\end{aligned}
$$

This gives

$$
\begin{aligned}
M_{g g \rightarrow H g}^{+++} & \simeq-\frac{64 m y \Delta}{u t}\left\{1-\frac{1}{2} m_{H}^{2} C\left(m_{H}^{2}\right)+\frac{1}{8} u t D(u, t)\right\} \\
& =\frac{8 y \Delta}{u t} M_{++}-8 m y \Delta D(u, t),
\end{aligned}
$$

and

$$
\begin{aligned}
M_{g g \rightarrow H g}^{++-} & \simeq \frac{64 m y \Delta}{u t}\left\{1-\frac{1}{2} m_{H}^{2} C\left(m_{H}^{2}\right)-\frac{1}{8} u t D(u, t)\right\} \\
& =-\frac{8 y \Delta}{u t} M_{++}-8 m y \Delta D(u, t) .
\end{aligned}
$$

In this regime, non-factorizing terms survive and give a logarithmically enhanced contribution, proportional to $\left(1 / p_{t}\right) \ln ^{2}\left(m^{2} / p_{t}^{2}\right)$ (see eq. (3.7)). We stress that those non-factorizing terms vanish for $p_{t}$ below $m$ (see e.g. eq. (3.5)), so that in the singular limit $p_{t} \rightarrow 0$ standard factorization of soft singularities is preserved. Furthermore, we argue that the non-factorizing terms above have a moderate impact on the squared amplitude. Indeed, in the amplitude squared the dominant contribution from $b$ quarks comes from the interference of bottom and top loops. For the top-loop 
contribution, $p_{t}^{2} \ll m_{t}^{2}$, the standard factorization holds. Since non-factorizing terms have opposite signs (with respect to factorizing ones) in $M_{g g \rightarrow H g}^{+++}$and $M_{g g \rightarrow H g}^{++-}$, see eqs. (3.8) and (3.9), they will cancel in the interference at this perturbative order. Non-vanishing terms in the amplitude squared appear in the pure $b$-quark amplitude squared and are of relative order $\left(m_{b} / m_{H}\right)^{4} \ln ^{4}\left(m_{b}^{2} / p_{t}^{2}\right)$ with respect to the dominant top contribution.

\subsection{Collinear limit}

We now consider the limit in which the emitted parton $p_{3}$ is collinear to e.g. $p_{1}$. Obviously, the same conclusions will apply to the case when $p_{3}$ is collinear to $p_{2}(u \leftrightarrow t)$. In this limit, the invariant $u$ vanishes, and, defining $z=m_{H}^{2} / s$, we have $t \rightarrow-(1-z) s$ and $u=-p_{t}^{2} /(1-z)$. Correspondingly the auxiliary invariants $s_{1}, t_{1}$ and $u_{1}$ become

$$
s_{1} \rightarrow-t, \quad t_{1} \rightarrow-s, \quad u_{1} \rightarrow-m_{H}^{2} .
$$

In this case we expect the amplitude squared to exhibit a collinear singularity $1 / u$. For simplicity, we consider the matrix element for the process $q g \rightarrow H q$, where $p_{1}$ and $p_{3}$ are the momenta of the incoming and outgoing quarks, respectively. Taking the collinear limit of the corresponding matrix element squared (see eq. (A.16)), we obtain

$$
\sum\left|M_{g q \rightarrow H q}\right|^{2} \simeq \frac{N_{c}^{2}-1}{2} \frac{\alpha_{s}^{3}}{\pi} \frac{1+(1-z)^{2}}{z}\left(\frac{1}{-z u}\right)|\mathcal{A}(u, t, s)|^{2},
$$

where $\mathcal{A}(u, t, s)$ is the contribution to the amplitude from a single quark in the loop, of mass $m$. In the collinear limit $u \rightarrow 0$, it is given by:

$$
\mathcal{A}(u, t, s) \simeq y m\left(2+4 m^{2} C_{1}(u)+u C(u)-m_{H}^{2} C\left(m_{H}^{2}\right)\right) .
$$

As before, we consider two regimes:

1. $p_{t}^{2} \ll m^{2} \ll m_{H}^{2}$. As in the soft case, from eq. (A.8) one sees that the triangle integral $u C(u)$ vanishes. We then obtain

$$
\mathcal{A}(u, t, s) \simeq 2 m^{2}\left(1-\frac{1}{2} m_{H}^{2} C\left(m_{H}^{2}\right)\right)=-\frac{m}{4} M_{++},
$$

which is proportional to the Born amplitude in eq. (2.7).

2. $m^{2} \ll p_{t}^{2} \ll m_{H}^{2}$. In this case $u C(u)$ does not vanish, and we have

$$
\begin{aligned}
\mathcal{A}(u, t, s) & \simeq 2 m^{2}\left(1-\frac{1}{2} m_{H}^{2} C\left(m_{H}^{2}\right)+\frac{1}{2} u C(u)\right) \\
& \simeq-\frac{m}{4} M_{++}+\frac{m^{2}}{2} \ln ^{2}\left(\frac{m^{2}}{-u}\right) .
\end{aligned}
$$

In this regime we do not have a factorization of the Born matrix element from the collinear singularity. Unlike in the soft case, there is no other amplitude against which this contribution can cancel, therefore a non-factorizing correction of order $\left(m_{b} / m_{H}\right)^{2} \ln ^{2}\left(p_{t} / m_{b}\right)$ remains in the amplitude. The presence of non-factorizing terms in the collinear limit has been noted also in ref. [30]. Similar contributions appear in the pure gluonic subprocess. 


\section{Factorization and resummation formula}

\subsection{Resummation formula}

In the previous section we have seen that, in the region $m^{2} \ll p_{t}^{2} \ll m_{H}^{2}$, the $\mathcal{O}\left(\alpha_{s}^{3}\right)$ matrix element for Higgs production with an extra jet is the sum of a factorizing and a non-factorizing term which vanishes in both the soft and collinear limits. The nonfactorizing term, not proportional to the Born matrix element, contains a new class of logarithms $\ln \left(p_{\mathrm{t}, \text { veto }} / m_{b}\right)$. At typical veto scales $p_{\mathrm{t}, \text { veto }}=25-30 \mathrm{GeV}$, these logarithms can be potentially as large as the factorizing Sudakov logarithms $\ln \left(m_{H} / p_{\text {t,veto }}\right)$ that we wish to resum. However, in the matrix element squared, their coefficients are suppressed by a factor $\left(m_{b} / m_{H}\right)^{2}$ in the collinear limit, and $\left(m_{b} / m_{H}\right)^{4}$ in the soft limit. Therefore it is important to investigate their actual numerical impact, and we will do so in the next subsection.

Since we do not know the structure of finite quark-mass corrections beyond $\mathcal{O}\left(\alpha_{s}^{3}\right)$, matching schemes need to preserve the structure of the perturbative series up to this order. The three multiplicative matching schemes defined in [6] and reported in appendix B automatically fulfill this structure. In these schemes, factorization is assumed to hold only for the singular terms containing $\ln \left(m_{H} / p_{\text {t,veto }}\right)$ which must be resummed since they become dominant in the low $p_{\mathrm{t} \text {,veto }}$ region. The additional terms (containing $\ln \left(m_{b} / p_{\text {t,veto }}\right)$ ) which vanish in the limit $p_{\mathrm{t} \text {,veto }} \rightarrow 0$ with $p_{\mathrm{t} \text {,veto }}>m_{b}$ are automatically treated as a regular remainder, which does not multiply the Born cross section.

Starting from this observation, we define our full NNLL resummed cross section for the jet veto as

$$
\begin{aligned}
& \Sigma_{\text {NNLL }}^{(J)}\left(p_{\text {t,veto }}\right)=\sum_{i, j} \int d x_{1} d x_{2}\left|M_{B}\right|^{2} \delta\left(x_{1} x_{2} s-M^{2}\right) \times \\
& \times\left[f_{i}\left(x_{1}, e^{-L} \mu_{F}\right) f_{j}\left(x_{2}, e^{-L} \mu_{F}\right)\left(1+\frac{\alpha_{s}}{2 \pi} \mathcal{H}^{(1)}\right)\right. \\
& \left.+\frac{\alpha_{s}}{2 \pi} \frac{1}{1-2 \alpha_{s} \beta_{0} L} \sum_{k} \int_{x_{1}}^{1} \frac{d z}{z}\left(C_{k i}^{(1)}(z) f_{i}\left(\frac{x_{1}}{z}, e^{-L} \mu_{F}\right) \times f_{j}\left(x_{2}, e^{-L} \mu_{F}\right)+\left\{\left(x_{1}, i\right) \leftrightarrow\left(x_{2}, j\right)\right\}\right)\right] \times \\
& \times\left(1+\mathcal{F}^{\text {clust }}+\mathcal{F}^{\text {correl }}\right) e^{L g_{1}\left(\alpha_{s} L\right)+g_{2}\left(\alpha_{s} L\right)+\frac{\alpha_{s}}{\pi} g_{3}\left(\alpha_{s} L\right)} .
\end{aligned}
$$

Here $L=\ln \left(Q / p_{\text {t,veto }}\right)$, where $Q$ is a resummation scale to be taken of order $m_{H}[3,6]{ }^{2}$ The coefficient functions $C_{k i}^{(1)}$, and resummation functions $g_{1}, g_{2}$ and $g_{3}$ are the same used in ref. [6]. The finite-mass effects in eq. (4.1) are contained both in the Born amplitude $\left|M_{B}\right|^{2}$ and in the one-loop coefficient $\mathcal{H}^{(1)}$ which accounts for the finite part of the virtual corrections to the Higgs production amplitude at order $\mathcal{O}\left(\alpha_{s}^{3}\right)$. The Born amplitude squared is related to its large- $m_{t}$ limit $\left|M_{B}^{\infty}\right|^{2}$ by the following relation

$$
\left|M_{B}\right|^{2}=\left|M_{B}^{\infty}\right|^{2}\left|\sum_{q} F_{0}\left(\tau_{q}\right)\right|^{2},
$$

\footnotetext{
${ }^{2}$ For all plots we actually replace $L$ by $\tilde{L}$ defined in appendix B. This ensures that the fixed order result is reproduced at high $p_{\mathrm{t}, \mathrm{veto}}$.
} 
where $\tau_{q}=m_{H}^{2} /\left(4 m_{q}^{2}\right)$ with $m_{q}$ denoting any heavy-quark mass. The function $F_{0}\left(\tau_{q}\right)$ is defined as $[10-12]$

$$
F_{0}\left(\tau_{q}\right)=\frac{3}{2 \tau_{q}^{2}}\left(\tau_{q}+\left(\tau_{q}-1\right) f\left(\tau_{q}\right)\right)
$$

where

$$
f\left(\tau_{q}\right)=\left\{\begin{array}{lr}
\arcsin ^{2}\left(\sqrt{\tau_{q}}\right), & \tau_{q} \leq 1 \\
-\frac{1}{4}\left[\ln \frac{\tau_{q}+\sqrt{\tau_{q}-1}}{\tau_{q}-\sqrt{\tau_{q}-1}}-i \pi\right]^{2}, & \tau_{q}>1
\end{array}\right.
$$

The one loop virtual corrections were computed in refs. [10-12]. In our resummation formula (4.1) they have the form

$$
\mathcal{H}^{(1)}=H^{(1)}-\left(B^{(1)}+\frac{A^{(1)}}{2} \ln \frac{m_{H}^{2}}{Q^{2}}\right) \ln \frac{m_{H}^{2}}{Q^{2}}+4 \pi \beta_{0} \ln \frac{\mu_{R}^{2}}{m_{H}^{2}},
$$

where

$$
H^{(1)}=2 c^{\mathrm{H}}\left(m_{t}, m_{b}\right)+\left(2+\frac{C_{A}}{2}\right) \pi^{2},
$$

where $\beta_{0}=\left(11 C_{A}-2 n_{f}\right) /(12 \pi)$ and $C_{A}=N_{c}$. All the remaining coefficients in eq. (4.4) are defined in ref. [6], while the expression we use for $c^{\mathrm{H}}\left(m_{t}, m_{b}\right)$ is taken from eq. (3.5) of ref. [12]. For the sake of simplicity we omit the explicit dependence of $\mathcal{H}^{(1)}$ and $H^{(1)}$ on the quark masses.

We obtain the fixed-order predictions from the program hnnlo $2.0[30],{ }^{3}$ in which the full NLO mass dependence is implemented and $\mathcal{O}\left(\alpha_{s}^{4}\right)$ corrections are computed in the large- $m_{t}$ limit and rescaled by the Born top-quark-mass correction factor $\left|F_{0}\left(\tau_{t}\right)\right|^{2}$ where $F_{0}\left(\tau_{t}\right)$ is defined in eq. (4.3).

\subsection{Treatment of non-factorizing terms}

In this section we want to study the numerical impact of mass-corrections motivating the validity of our resummation formula eq. (4.1) and matching schemes eqs. (B.2), (B.6) and (B.7). We also study the impact of non-factorizing terms and give a procedure to estimate the uncertainty associated to them.

Since we can compute mass-effects exactly only to first order in perturbation theory, we study the impact of mass-effects on the so-called remainder function. The remainder function is obtained from the fixed-order result, once all divergent logarithms are subtracted at the given order in perturbation theory. In our case, we treat all logarithms of the form $\ln \left(p_{t} / m_{H}\right)$, that can be resummed using standard techniques, as divergent, and hence we subtract them from the fixed order to obtain the remainder function. ${ }^{4}$ For bottom induced production, defined as both the top-bottom interference and the bottom squared contribution, the remainder function will still contain logarithms of the form $\ln \left(p_{t} / m_{b}\right)$ for $p_{t}>m_{b}$, which vanish when $p_{t} \rightarrow 0$. Nevertheless, even if these logarithms vanish for $p_{t} \rightarrow 0$ one should keep in mind that additional large logarithms of the ratio

\footnotetext{
${ }^{3}$ We wish to thank M. Grazzini for providing us with a preliminary version of this code.

${ }^{4}$ More precisely, we subtract $\tilde{L}$ terms defined in eq. (B.3), rather then $L$.
} 


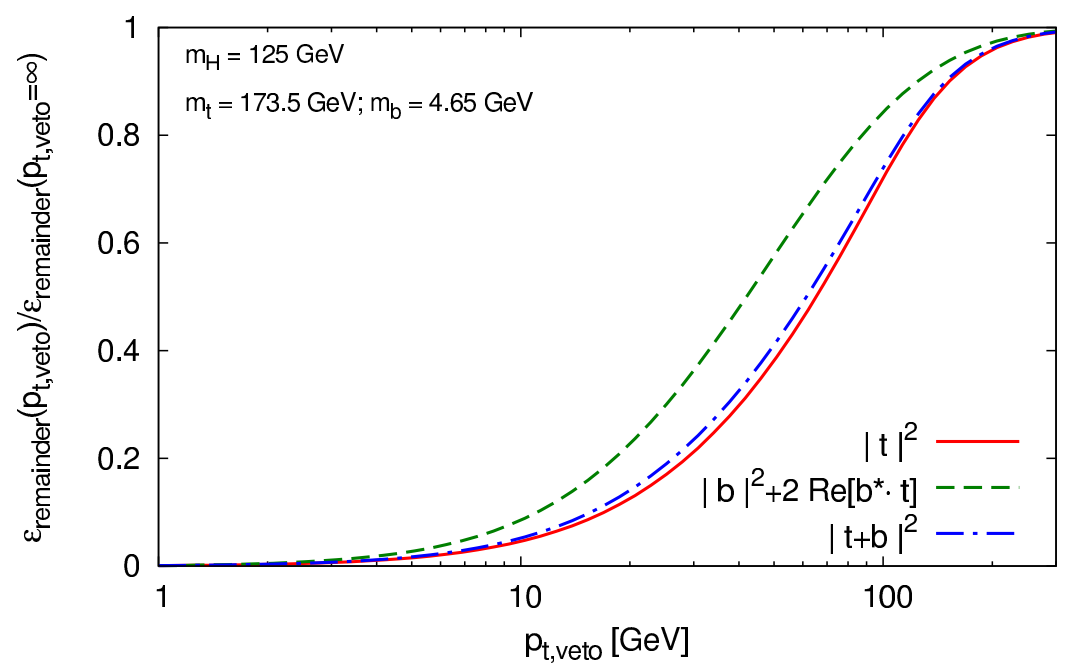

Figure 1. Regular terms in the jet veto efficiency including top-quark effects (solid red), bottomquark and interference effects (dashed green) and full top- and bottom-quark effects (dashed blue). Each curve is normalized to its asymptotic value at large $p_{\mathrm{t}, \mathrm{veto}}$.

$m_{b} / m_{H}$ are present in the virtual corrections eq. (4.4) (see e.g. eq. (44) of [10]). At finite $p_{\text {t,veto }}$ logarithms $\ln ^{2}\left(m_{b} / m_{H}\right)$ are also present in the remainder function. Since we do not know the higher-order structure of such terms, in the following we will propose various ways to estimate their impact.

In figure 1 we show the remainder contributions to the jet-veto efficiency including topquark effects only (solid, red), bottom-induced (dashed, green) and full top- and bottomquark effects (dot-dashed, blue). Each curve is normalized to its asymptotic value at large $p_{t, \text { veto }}$, this is because we are interested in studying the departure from the infrared $\left(p_{\mathrm{t}, \text { veto }} \rightarrow 0\right)$ limit. From the plot it is evident that the bottom induced contribution (dashed, green) rises faster than the pure top contribution (solid, red). It is however also clear that once all effects are taken into account (dot-dashed, blue) the difference with respect to the top only contribution is modest. This is expected, since the top contribution is dominant. The faster rise of the bottom induced contribution can be interpreted as an indication that the resummation starts being unreliable at a lower $p_{\mathrm{t} \text {,veto value, meaning }}$ that a lower resummation scale should be used for the bottom-induced contribution. Similarly to what has been done in ref. [30] we can then introduce two different resummation scales: $Q_{1}$ for the top contribution and $Q_{2}$ for the bottom-induced one. We show then in figure 2 the impact of varying $Q_{2}$ in our default matched formula, eq. (B.2) with default settings as defined in ref. [6] and recalled at the beginning of section 5 , from values as low as the bottom mass (we choose $m_{H} / 25$ ) to values as high as half the Higgs mass.

Figure 2 (left) shows the effect of $Q_{2}$ variation on the pure bottom-induced contribution, while keeping all other scales and settings to their default values. In practice, we obtain the bottom-induced contribution by subtracting the top-only correction from the full result which includes both top and bottom effects. We take values of $Q_{2}$ equal to $m_{H} / 25, m_{H} / 8, m_{H} / 4$, and $m_{H} / 2$. We see that, as expected, two curves obtained 

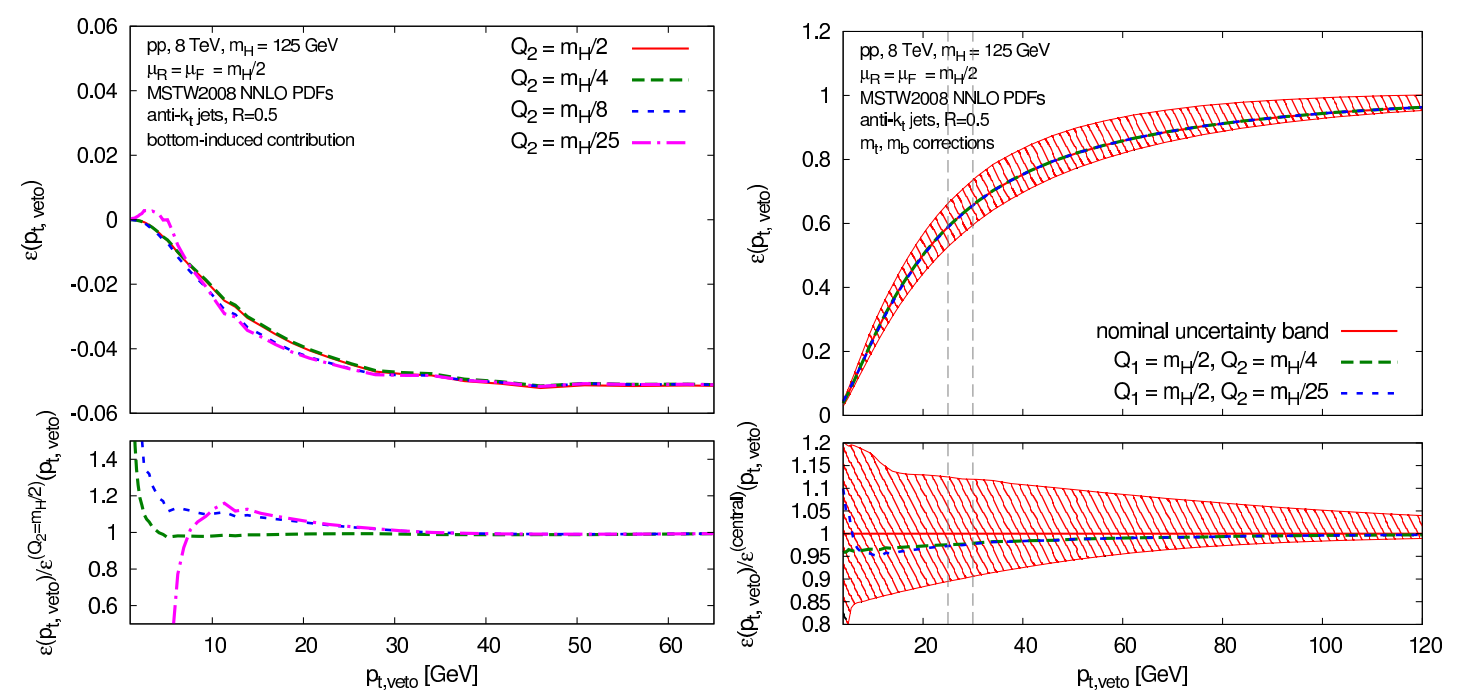

Figure 2. The effect of varying $Q_{2}$, while keeping all our scales and settings to their default values, on the bottom-induced contribution (left) and on the total resummed efficiency (right).

respectively with $Q_{2}=Q_{a}$ and $Q_{2}=Q_{b}$ are in good agreement for $p_{\text {t,veto }}>\max \left(Q_{a}, Q_{b}\right)$. Furthermore, all curves agree above $p_{\mathrm{t} \text {,veto }}=30-40 \mathrm{GeV}$, indicating that the resummation of bottom-induced corrections becomes negligible in this region. Figure 2 (right) shows the full resummed efficiency including both top and bottom-induced corrections. We see that the curves obtained with $Q_{2}=m_{H} / 25$ or $Q_{2}=m_{H} / 4$ (while keeping again all our settings to their default values) lie within our nominal uncertainty band, as defined at the beginning of section 5 and shown as a red band in figure 2 (right). In particular, we notice a decrease of the order of $2-3 \%$ in the central value at $p_{\mathrm{t} \text {,veto }}=25-30 \mathrm{GeV}$ when taking a lower $Q_{2}$ value. Furthermore, we see that all predictions lie on top of each other

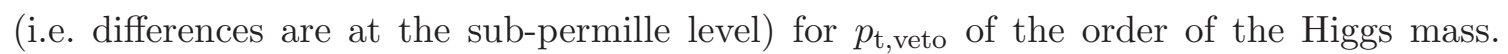
This is expected since our matching formulae guarantee that at high values of $p_{\mathrm{t} \text {,veto our }}$ predictions reproduce the fixed order result.

Another possible way to estimate the impact on the final result of missing higher orders is to consider also a matching scheme in which the whole one-loop remainder is exponentiated. Matching schemes where these terms are naturally exponentiated belong to the so called $\log R$ matching-scheme category [34]. We define a hybrid matching scheme, where the one-loop remainder is exponentiated whilst the two-loop contribution is treated as done in scheme (a). We refer to this scheme as "logR-(a)", it is defined more precisely in appendix B. We then use the difference between our default matching scheme "a" and the "logR-(a)" matching scheme, to assess the uncertainty due to bottom-induced mass effects.

Figure 3 (left) shows the difference between our nominal uncertainty band (defined in section 5) and an uncertainty band associated to $\log \mathrm{R}$-(a) scheme. The latter is obtained by performing the same scale variations as for the nominal band, while keeping the matching scheme fixed. We see that at $p_{\mathrm{t} \text {,veto }}=25-30 \mathrm{GeV}$ the central value is increased by about $2-3 \%$ but it lies well within the nominal uncertainty band. We also note that 

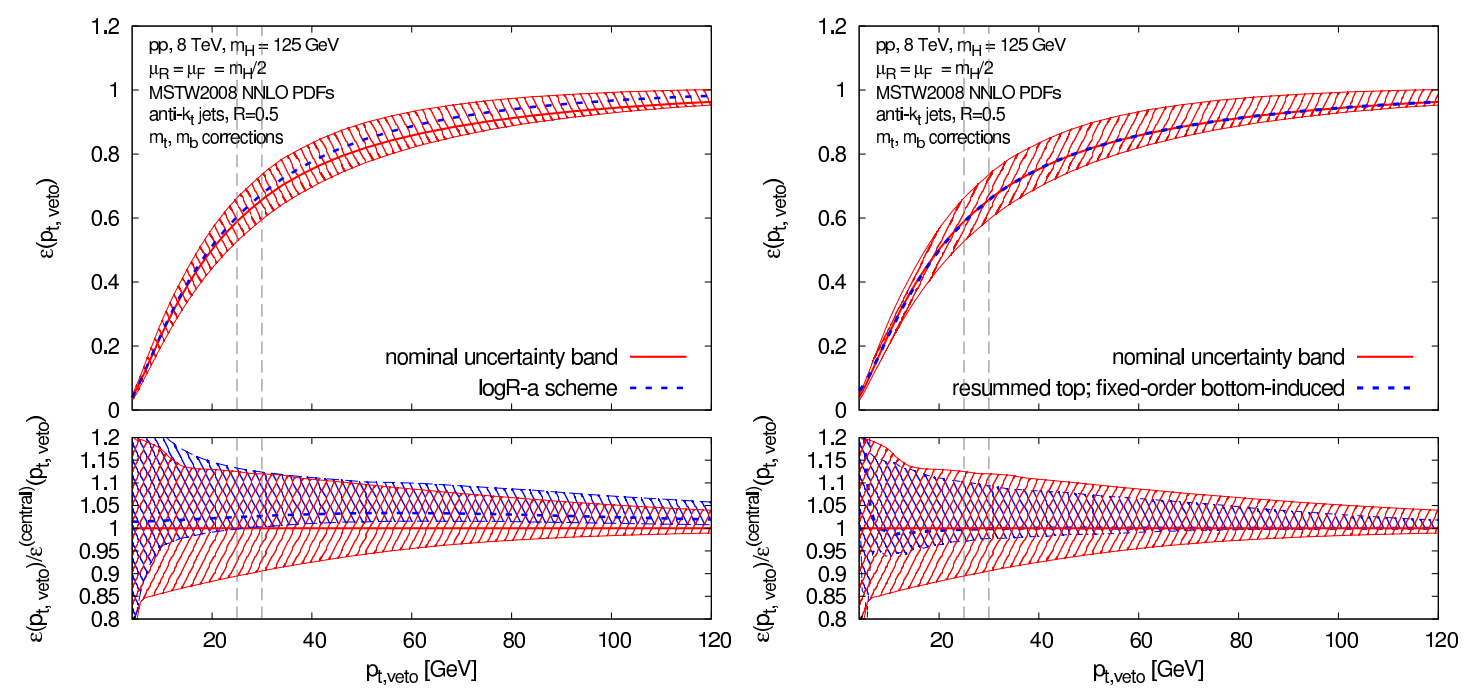

Figure 3. The left plot shows a comparison between our nominal uncertainty band (red) and the one obtained using the logR-(a) matching scheme (blue). See text for more details. The right plot shows the same nominal uncertainty band (red) compared to a prediction in which only the top-quark contributions are resummed, while keeping the whole bottom-induced contribution at fixed-order (blue). The uncertainty band in the latter case is obtained by varying renormalization, factorization and resummation scales.

the $\log \mathrm{R}$-(a) band is smaller than the nominal band. This is not surprising since the latter band additionally accounts for matching scheme uncertainties. In particular, the lower edge of the nominal band is driven by matching scheme (b). Consistently with what we have done in the large- $m_{t}$ case $[3,6]$, we could add the central value of the $\log \mathrm{R}$-(a) scheme band to our nominal band. However, in practice this does not make any difference since it is fully included in the original nominal band.

The right plot of figure 3 shows a comparison between the nominal uncertainty band and a conservative prediction in which only the top-quark contributions are resummed to NNLL, while the bottom-induced contributions are simply added at fixed-order as obtained with the generator hnnlo 2.0. The blue uncertainty band in the latter case is obtained as the envelope of the variation of renormalization and factorization scales in both the fixedorder and the resummed terms and the resummation scale in the resummed contribution. Also in this case we do not observe sizable differences between the two central values and the resulting uncertainty band is fully compatible with our nominal prediction.

In a similar way, the effect of "factorization breaking" above $m_{b}$ can be studied by introducing a modified (a) matching scheme, mod-(a), in which the one-loop remainder is treated additively, rather than being multiplied by a Sudakov factor. We find that the numerical difference from scheme (a) is negligible. A detailed discussion is reported in appendix C.

We therefore conclude that the method we have used to assess the theory uncertainties in the large- $m_{t}$ case is robust and does not need to be modified when mass-effects are taken into account. 


\section{Phenomenology}

In the present section we study the phenomenological impact of heavy-quark mass corrections on the jet-veto efficiency and cross section. We consider $8 \mathrm{TeV}$ LHC collisions and the production of a Higgs boson of mass $m_{H}=125 \mathrm{GeV}$ and set the top and bottom masses to $m_{t}=173.5 \mathrm{GeV}$ and $m_{b}=4.65 \mathrm{GeV}$, respectively. Throughout our analysis we use the PDF set MSTW2008NNLO [35] and the anti- $k_{t}$ jet algorithm [9] with $R=0.5$, as implemented in FastJet $[36,37]$ to reconstruct jets.

As detailed in the previous section, we match the NNLL resummed cross section to the fixed-order NNLO prediction using the three matching schemes (B.2), (B.6), and (B.7). We remark that the matching schemes used here differ in one important aspect from the ones used in the large- $m_{t}$ case in ref. [6]. In fact, the expansion at relative order $\mathcal{O}\left(\alpha_{s}^{2}\right)$ appearing in the matching formulae is obtained from the large- $m_{t}$ resummation formula [6], rescaled by the Born top-quark-mass correction factor $\left|F_{0}\left(\tau_{t}\right)\right|^{2}$ where $F_{0}\left(\tau_{t}\right)$ is defined in eq. (4.3). This ensures that the matching procedure does not spoil the NNLL accuracy of our resummation in the small $p_{\mathrm{t} \text {,veto }}$ limit. However, this also implies that the difference between matched and fixed-order results remains of relative order $\mathcal{O}\left(\alpha_{s}^{2}\right)$, and not $\mathcal{O}\left(\alpha_{s}^{3}\right)$ as one would expect from a NNLO matching. The uncertainties in the jet-veto efficiency and in the zero-jet cross section are assessed as previously discussed in refs. [3, 6] and as recalled here. The default central prediction is obtained by setting renormalization $\mu_{R}$, factorization $\mu_{F}$ and resummation scale $Q$ all equal to half the Higgs mass, $\mu_{F}=\mu_{R}=Q=m_{H} / 2$, and matching scheme (a), eq. (B.2). Uncertainties are obtained by varying both $\mu_{F}$ and $\mu_{R}$ by a factor of two in either direction, keeping $1 / 2 \leq \mu_{R} / \mu_{F} \leq 2$. Moreover, using central values for $\mu_{R}$ and $\mu_{F}$, we vary the resummation scale $Q$ by a factor of two in scheme (a) and compute the central values of matching schemes (b), eq. (B.6) and (c), eq. (B.7). The final uncertainty for the matched prediction is obtained as the envelope of all these variations.

Numerical results for the jet-veto efficiency are shown in the left plot of figure 4 . In the region of interest for $\mathrm{LHC}$ analyses, i.e. $p_{\mathrm{t} \text {,veto }}$ in the range $25-30 \mathrm{GeV}$, the matched prediction for the efficiency is $2-3 \%$ higher than the fixed-order one. The corresponding theoretical uncertainty is reduced by roughly a factor of two when the resummation is included. The right plot of figure 4 shows the comparison between the NNLL+NNLO exclusive zero-jet cross section in the large- $m_{t}$ limit and the one including the full top- and bottom-quark mass dependence. We see that the integrated cross section is about $7 \%$ larger if the full top-quark mass dependence is included (quite independently of the value of $p_{\mathrm{t}, \mathrm{veto}}$ ), whereas it is $1-2 \%$ lower for $p_{\text {t,veto }}=25-30 \mathrm{GeV}$, when also the bottom is taken into account.

In figure 5 we show the jet-veto efficiency computed at NNLL+NNLO with and without exact mass dependence. We observe an increase of $2-3 \%$ in the uncertainty at $p_{\mathrm{t} \text {,veto }}=25-30 \mathrm{GeV}$ when the bottom-induced contributions are included, compared to both the large and the exact $m_{t}$ results. This is due to the larger remainder function which pushes the schemes (b) and (c) results to lower and higher values, respectively. As a consequence, the uncertainty band in the presence of full mass corrections is totally driven by the central values obtained with schemes (b) and (c) which constitute the lower and upper edges of the band, respectively. This is in contrast with what happens both 

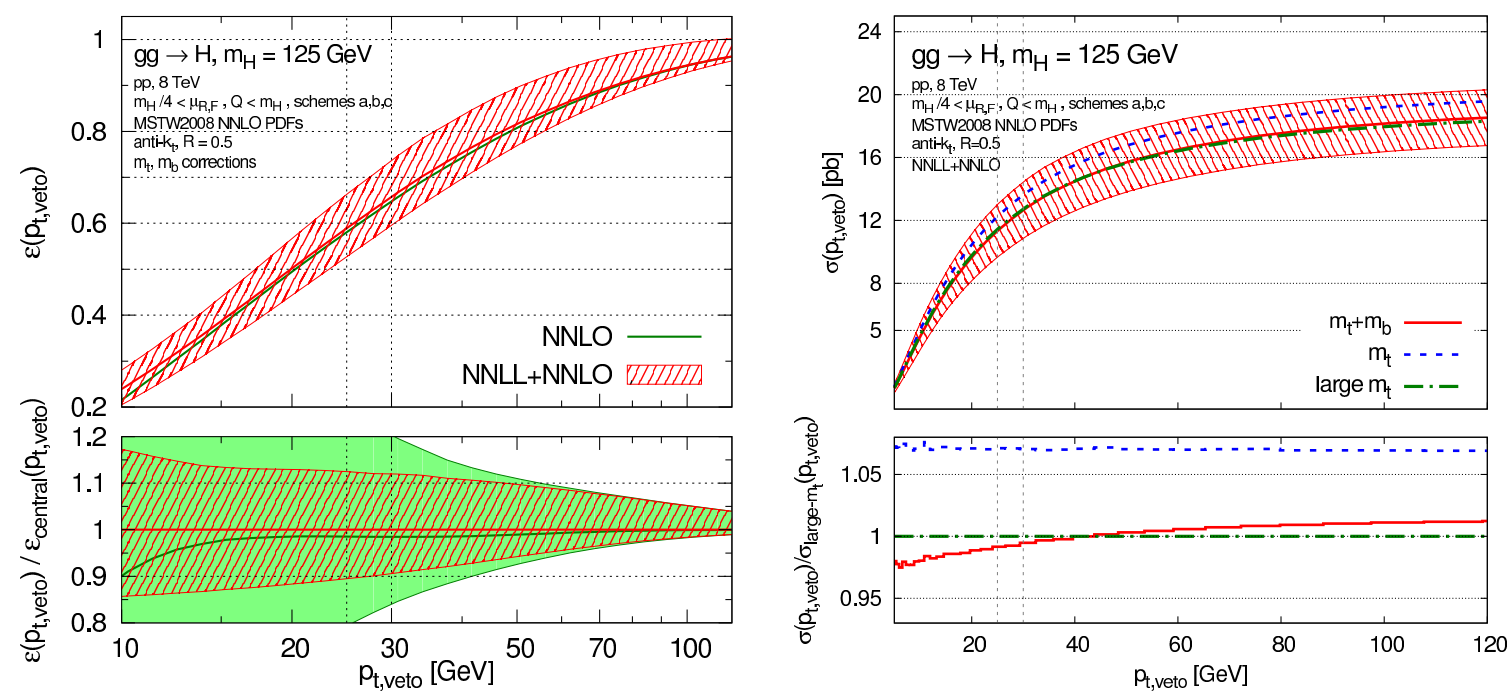

Figure 4. Left: comparison between the matched efficiency at NNLL+NNLO accuracy and the NNLO fixed-order result, both results include mass effects. Right: zero-jet cross section computed in the large- $m_{t}$ limit (green dot-dashed curve), including full $m_{t}$ dependence (blue dashed curve), and including full $m_{t}$ and $m_{b}$ dependence (red solid band).

Exact $\mathrm{m}_{\mathrm{t}}$ and $\mathrm{m}_{\mathrm{b}}$ corrections

\begin{tabular}{|c|c|c|c|c|}
\hline $\mathrm{R}$ & $p_{\text {t,veto }}[\mathrm{GeV}]$ & $\sigma_{\text {tot }}^{8 \mathrm{TeV}}[\mathrm{pb}]$ & $\epsilon^{(8 \mathrm{TeV})}$ & $\sigma_{0 \text {-jet }}^{(8 \mathrm{TeV})}[\mathrm{pb}]$ \\
\hline 0.4 & 25 & $19.24 \pm 1.78$ & $0.602 \pm 0.070$ & $11.59 \pm 1.72$ \\
0.5 & 30 & $19.24 \pm 1.78$ & $0.657 \pm 0.070$ & $12.64 \pm 1.79$ \\
\hline
\end{tabular}

Table 1. Total inclusive cross-section, jet-veto efficiency and zero-jet cross-section for Higgs pro-

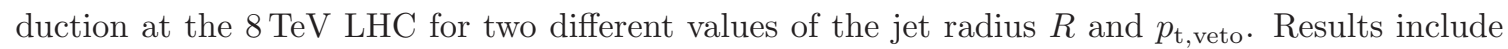
exact top and bottom mass dependence. The quoted total cross section and the corresponding errors have been computed with the hnnlo 2.0 code [38].

in the large- $m_{t}$ limit and if only $m_{t}$ corrections are included where the upper edge of the band is driven by the resummation scale variation.

Below we provide tables with numerical results for cross-sections and efficiencies for the values of veto scales and jet radii, as used in current LHC analyses. All uncertainties have been symmetrized with respect to the central value.

We first examine only the first three tables in which the total cross-section is computed at NNLO QCD. We notice that top-mass effects increase the central values by about 6-7\%. Bottom mass effects bring the central values down again, leading to a modest decrease of about half a percent in the zero-jet cross-section with respect to the large- $m_{t}$ result. As already noticed earlier, we see that the uncertainties in the cross-section remain unchanged when including only top mass effects, while they increase by about $2 \%$ (and amount to about 14\%) when also bottom-induced corrections are taken into account. By comparing with figure 2 of ref. [6] we note that when mass corrections are taken into 


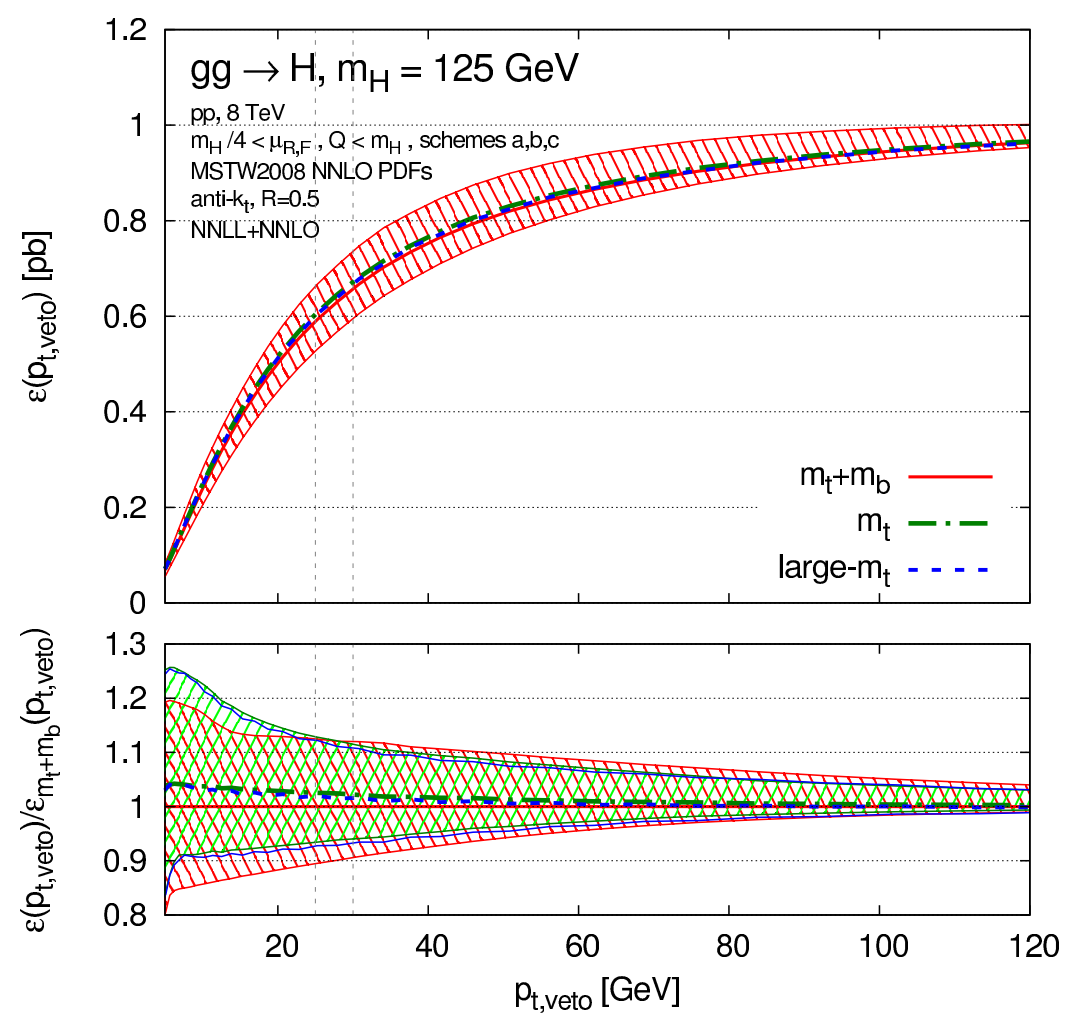

Figure 5. Comparison between the matched efficiency at NNLL+NNLO accuracy in the large- $m_{t}$ limit (dashed blue), including $m_{t}$ only (dot-dashed green) and both $m_{t}, m_{b}$ effects (solid red). The lower panel shows the ratio to the central value of the $m_{t}+m_{b}$ band.

Exact $\mathbf{m}_{\mathrm{t}}$ corrections only

\begin{tabular}{|c|c|c|c|c|}
\hline $\mathrm{R}$ & $p_{\text {t,veto }}[\mathrm{GeV}]$ & $\sigma_{\text {tot }}^{8 \mathrm{TeV}}[\mathrm{pb}]$ & $\epsilon^{(8 \mathrm{TeV})}$ & $\sigma_{0 \text {-jet }}^{(8 \mathrm{TeV})}[\mathrm{pb}]$ \\
\hline 0.4 & 25 & $20.16 \pm 1.87$ & $0.617 \pm 0.063$ & $12.44 \pm 1.72$ \\
0.5 & 30 & $20.16 \pm 1.87$ & $0.672 \pm 0.057$ & $13.54 \pm 1.71$ \\
\hline
\end{tabular}

Table 2. As table 1 but including only exact top mass dependence.

\section{Large- $\mathbf{m}_{\mathrm{t}}$ approximation}

\begin{tabular}{|c|c|c|c|c|}
\hline $\mathrm{R}$ & $p_{\text {t,veto }}[\mathrm{GeV}]$ & $\sigma_{\text {tot }}^{8 \mathrm{TeV}}[\mathrm{pb}]$ & $\epsilon^{(8 \mathrm{TeV})}$ & $\sigma_{0 \text {-jet }}^{(8 \mathrm{TeV})}[\mathrm{pb}]$ \\
\hline 0.4 & 25 & $19.03 \pm 1.76$ & $0.613 \pm 0.064$ & $11.66 \pm 1.62$ \\
0.5 & 30 & $19.03 \pm 1.76$ & $0.667 \pm 0.058$ & $12.70 \pm 1.61$ \\
\hline
\end{tabular}

Table 3. As table 1 but in the large $m_{t}$ approximation.

account, the uncertainty in the resummed prediction increases by about $2 \%$, while the fixed order uncertainty increases from about $15-20 \%$ to about $20-25 \%$. 
Large- $\mathbf{m}_{\mathbf{t}}$ approximation $\left(\sigma_{\mathrm{tot}}^{8 \mathrm{TeV}}\right.$ from HXSWG)

\begin{tabular}{|c|c|c|c|c|}
\hline $\mathrm{R}$ & $p_{\text {t,veto }}[\mathrm{GeV}]$ & $\sigma_{\text {tot }}^{8 \mathrm{TeV}}[\mathrm{pb}]$ & $\epsilon^{(8 \mathrm{TeV})}$ & $\sigma_{0 \text {-jet }}^{(8 \mathrm{TeV})}[\mathrm{pb}]$ \\
\hline 0.4 & 25 & $19.27 \pm 1.45$ & $0.613 \pm 0.064$ & $11.81 \pm 1.51$ \\
0.5 & 30 & $19.27 \pm 1.45$ & $0.667 \pm 0.058$ & $12.86 \pm 1.47$ \\
\hline
\end{tabular}

Table 4. As table 1 but in the large $m_{t}$ approximation. Unlike in table 3 the total cross-section is taken from the HXSWG [39] and includes finite-width, electro-weak and threshold resummation effects.

In table 4 we report the jet-veto efficiency and cross-section using the improved total cross-section recommended by the Higgs Cross section Working Group (HXSWG) [39] instead of the pure NNLO value. Improvements include the treatment of the Higgs width, NNLL threshold effects and NLO electro-weak corrections. To figure out only the size of mass effects one has to compare results including mass corrections to table 3 , rather than table 4 . The improved predictions for the total cross section included in table 4 are not available when finite-mass effects are included. The difference between tables 3 and 4 can then help estimate the effect of these higher-order corrections on the results of tables 1 and 2 .

All results presented so far used $R=0.5$ as a jet-radius to define jets. As noticed first in ref. [6], when the Higgs is produced in the large- $m_{t}$ limit the uncertainty band is reduced when using a larger radius. This is because in the latter case the upper edge of the uncertainty band is determined by resummation scale dependence of the NNLL corrections which are smaller at larger values of $R$. As we pointed out previously, when we include the exact mass dependence the uncertainty band is fully determined by the spread between matching schemes (b) and (c). This spread is not expected to decrease when larger values of $R$-values are considered, this is why we do not observe any reduction in the uncertainty when considering a larger radius (see figure 6).

Since mass effects are also implemented in NLO Monte Carlo generators, POWHEG [27, $40,41]$ and MC@NLO $[28,42]$, it is instructive to carry out a comparison to their predictions. The comparison is particularly interesting since the two event generators have a different treatment of heavy-quark mass corrections. In particular, in POWHEG mass corrections are included in the Sudakov form factor, while in MC@NLO they are treated as finite remainders. The right plots of figures 7 and 8 show four different predictions for the ratio of the leading-jet $p_{t}$ distribution, normalized to the corresponding total cross section, to the same distribution in the large- $m_{t}$ approximation, as obtained from JetVHeto at NNLL+NNLO (red, solid), at NNLO (green, dot-dashed), POWHEG+Pythia (blue, dashed) and MC@NLO+Herwig (red, dashed). All Monte Carlos are run at parton level only, with no multi-parton interactions or hadronization corrections. For completeness, the comparison to NLL+NLO and NLO is reported in the left plots of figures 7 and 8 .

We see that the three predictions for the ratio agree well if only the top-quark is included (figure 7). At high $p_{\text {t,veto }}$ JetVHeto differs from the NLO Monte Carlo predictions in the right plots. This is not surprising since JetVHeto is NLO (rather then LO) accurate 

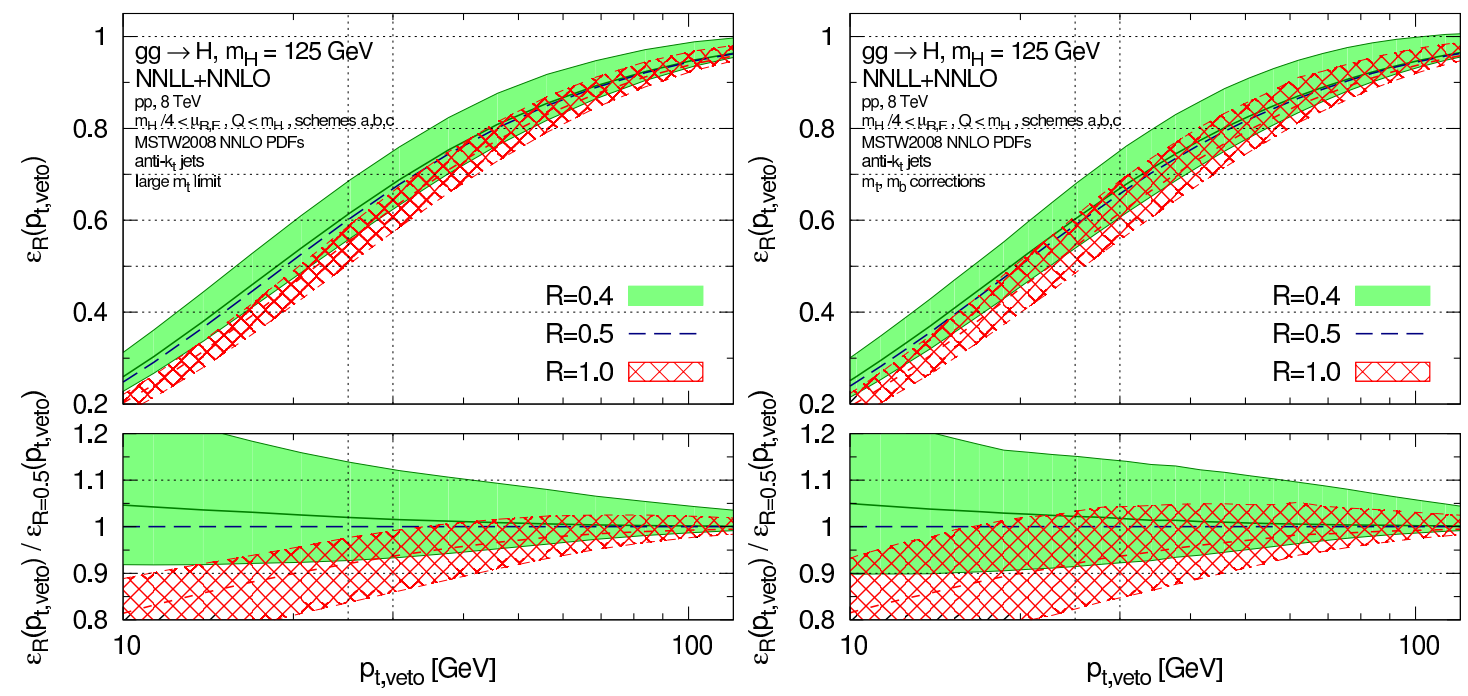

Figure 6. Comparison between the matched efficiencies at NNLL+NNLO accuracy for different values of the jet radius in the large- $m_{t}$ limit (left) and with $m_{t}, m_{b}$ effects (right). The lower panel shows the ratio to the central value with $R=0.5$.
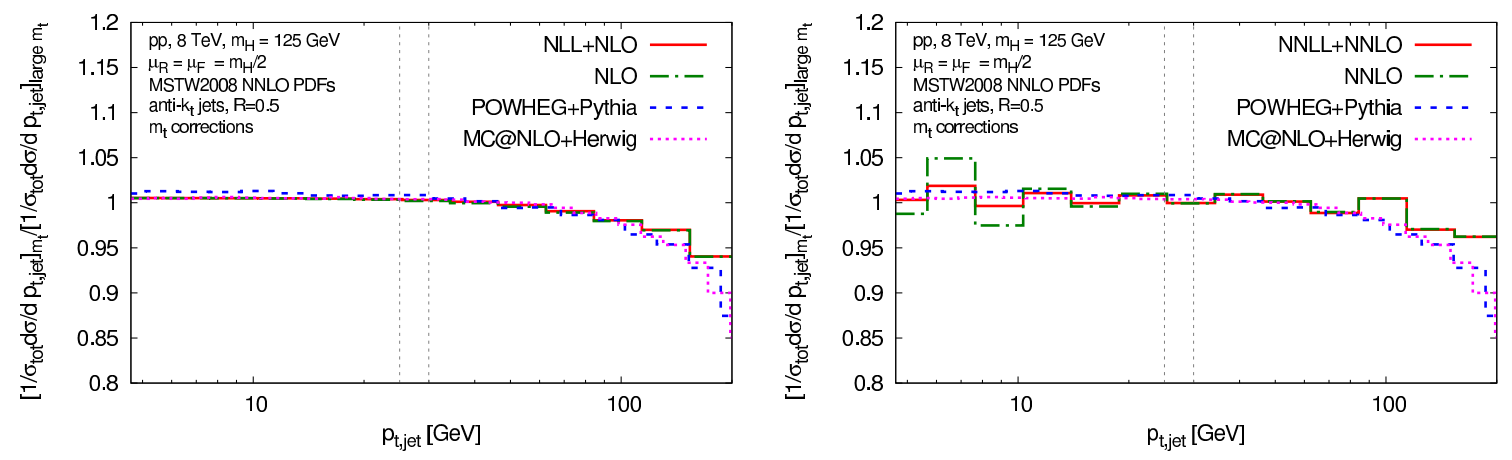

Figure 7. Ratios of the leading-jet $p_{t}$ distribution (normalized to the total cross section) including full dependence on the top mass, to the same distribution in the large- $m_{t}$ approximation (also normalized). In the plots labelled NNLL+NNLO and NNLO, mass corrections are included only at NLO, as described in the text.

in the jet-veto spectrum. On the contrary, when bottom-quark effects are included (figure 8), predictions differ over the whole spectrum.

In general we find that in this case the prediction from JetVHeto lies somewhat in between that of POWHEG+Pythia and MCONLO+Herwig, but tends to be closer to the latter. In particular, at usual veto scales, $25 \mathrm{GeV} \leq p_{\text {t,veto }} \leq 30 \mathrm{GeV}$, we found better agreement with MC@NLO. Compared to JetVHeto, POWHEG seems to enhance the size of $m_{b}$ effects, while MC@NLO seems to diminish them.

Finally, it is interesting to verify whether Monte Carlo predictions lie in the uncertainty band of JetVHeto for the efficiency. Figure 9 shows the predictions for the jet-veto efficiency obtained with JetVHeto, with its uncertainty band, POWHEG, MC@NLO and HJ-MiNLO $[43,44]$ (the latter uses the large $m_{t}$ approximation). We find that for $p_{\text {t,veto }}>20 \mathrm{GeV}$ all predictions lie in the uncertainty band of JetVHeto. In fact, 

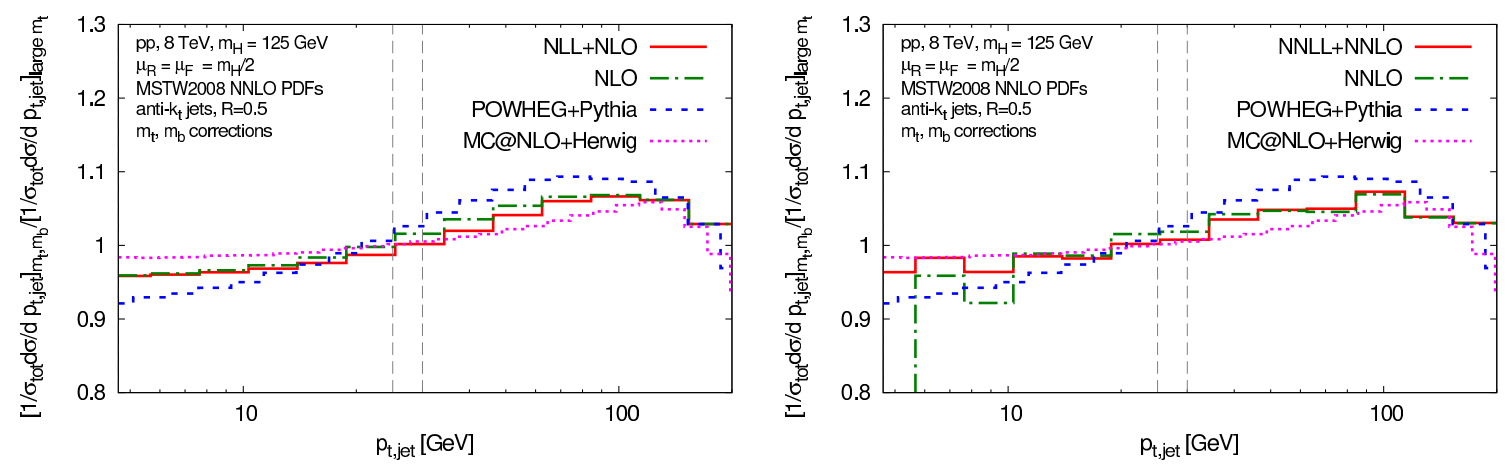

Figure 8. As in figure 7 including dependence on top and bottom masses.

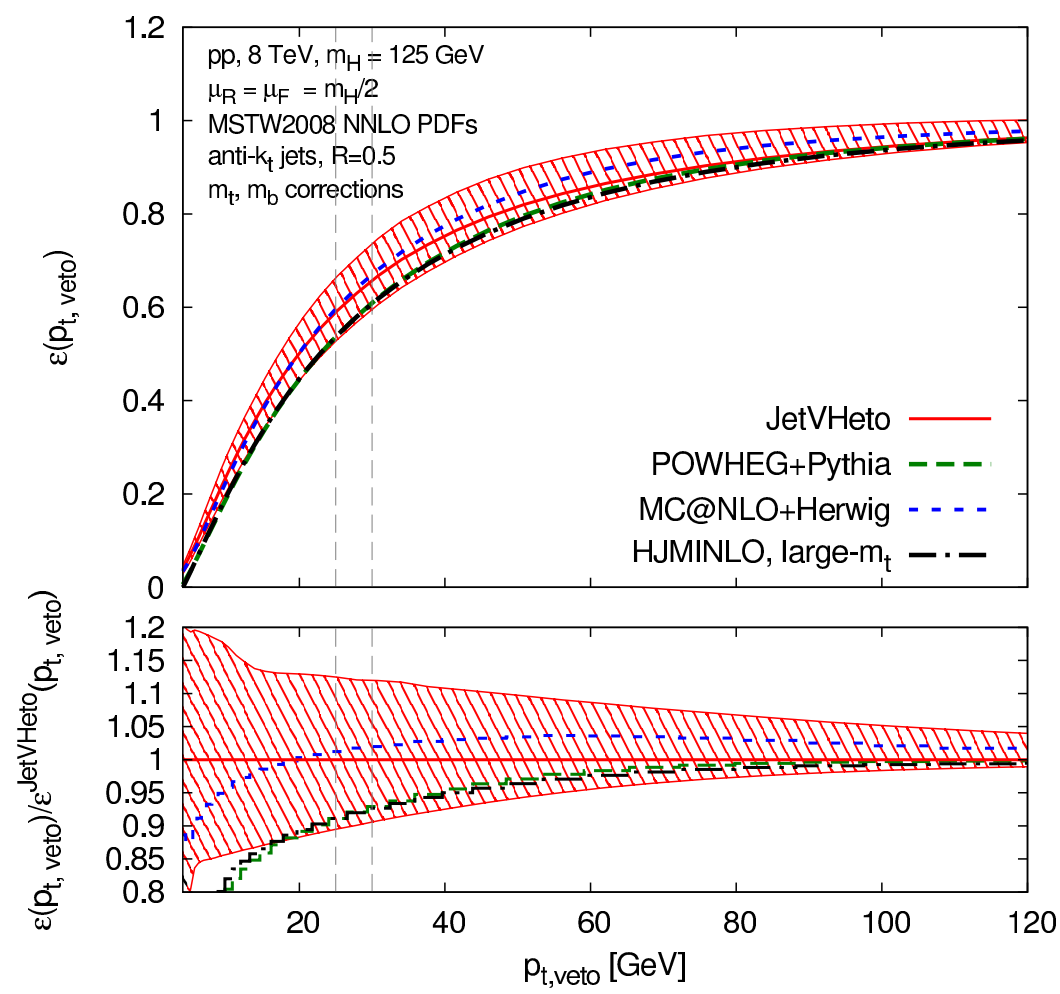

Figure 9. Comparison between different event generators for the jet-veto efficiency.

POWHEG+Pythia tends to the central value of the JetVHeto predictions at high $p_{\text {t,veto }}$, while MC@NLO is closer to JetVHeto at lower $p_{\text {t,veto }}$.

\section{Conclusions}

In the present work we studied the size of finite-mass effects in the resummed jet-veto efficiency and zero-jet cross section for Higgs-boson production. The inclusion of these corrections is not trivial since the mass of virtual quarks introduce additional scales in the problem, besides the Higgs mass and the jet-veto scale. In particular, when the bottom 
quark is included, new non-factorizing logarithms of the type $\ln \left(p_{\mathrm{t}, \mathrm{veto}} / m_{b}\right)$ appear if the emitted final state partons resolve the quark loop, i.e. in the kinematical range $m_{b}<p_{\text {t,veto }}$.

Since such new logarithms vanish for $p_{\text {t,veto }}<m_{b}$, we argue that it is reasonable to treat them as any regular remainder. We have validated our resummation and matching procedure by varying the resummation scale related to the bottom-induced terms, and by exponentiating the one-loop remainder using a logR-type matching scheme. Still, a two-loop result of mass-effects would be useful to get an insight into the structure of non-factorizing terms to higher orders.

As already observed in ref. [29] for the Higgs transverse momentum distribution, we also find that bottom-mass effects distort the jet-veto distribution. However, we also find that bottom-quark effects are as large as finite top corrections and opposite in sign, so that this destructive interference leads to very small effects for jet-veto values currently used at the LHC. Compared to the large $m_{t}$ limit the jet-veto efficiency (zero-jet cross-section) decreases by about $1.5 \%(0.5 \%)$ for $p_{\mathrm{t} \text {,veto }}=25-30 \mathrm{GeV}$, when both top and bottom effects are taken into account. The uncertainty however increases by about $2 \%$ with respect to the large- $m_{t}$ limit. Unlike what observed in ref. [30], we find that our predictions are stable when varying the resummation scale $Q_{2}$ of the bottom-induced contribution, also at high values of $Q_{2}$. Furthermore, we find modest corrections even at very small transverse momentum values (we stress however that we are looking at different observables).

Given the very small effects that we find here, it is natural to wonder why mass-effects in POWHEG or HRes, as implemented in ref. [30] and [45], give rise to much larger corrections at low $p_{t}$. In order to investigate this issue, we have performed a NNLL+NNLO calculation for $p_{t H}$ as well, including mass effects as described in the present paper. Our results are given in appendix D. We find that, if we match resummed results to NLO distributions, in the intermediate $p_{t}$ region, between 20 and $50 \mathrm{GeV}$, the matched distributions differ substantially from the corresponding fixed-order predictions when choosing a standard resummation scale of the order of teh Higgs mass. This is true both in the large- $m_{t}$ limit and including finite-mass effects (top and bottom contributions). On the other hand, when the matching is performed at NNLO, we find a much better agreement between matched and fixed-order distributions, more details are given in appendix D. We note that ref. [30] considers a matching to NLO only for the bottom-induced contribution. Insofar, our results are not in disagreement with that reference.

The effects that we find here are very modest, because of both the Yukawa suppression for bottom quarks and of the destructive interference. When the leading jet has a transverse momentum that is much larger than the top mass, as in boosted studies, logarithms of the form $\ln \left(p_{t} / m_{t}\right)$ can be potentially large and might need to be resummed to all orders. We stress that these mass effects are not treated properly by Monte Carlo generators. Moreover, the non-factorizing logarithms are not resummed by any parton shower. When integrated over the whole $p_{\mathrm{t} \text {,veto }}$ spectrum, such logarithms give rise to terms of the form $\ln \left(m_{H} / m\right)$ in the total cross section which can be sizable in heavy-Higgs searches.

Our formulae have been implemented in the public code JetVHeto [46], distributed together with a number of benchmark results. 


\section{Acknowledgments}

We particularly thank Paolo Nason and Gavin Salam for extensive discussions. We also acknowledge useful discussions with Mrinal Dasgupta, Keith Hamilton and Paolo Torrielli. We thank ESI (all) and KITP (GZ) for hospitality while part of this work was carried out. GZ is supported by Science and Technology Facility Council. PFM is supported by the Swiss National Science Foundation (SNF) under grant 200020-138206. AB is supported by the Science Technology and Facilities Council (STFC) under grant number ST/J000477/1.

\section{A Real emission matrix elements}

We report here the lowest order matrix elements including finite mass effects for the production of a Higgs boson in association with a (anti-)quark or a gluon. These are taken directly from ref. [33].

We denote by $p_{1}$ and $p_{2}$ the momenta of the incoming partons and with $p_{3}$ that of the outgoing parton. We express the matrix elements in terms of the usual Mandelstam invariants

$$
s=\left(p_{1}+p_{2}\right)^{2}, \quad t=\left(p_{2}-p_{3}\right)^{2}, \quad u=\left(p_{1}-p_{3}\right)^{2},
$$

and of the auxiliary invariants

$$
s_{1}=s-m_{H}^{2}, \quad t_{1}=t-m_{H}^{2}, \quad u_{1}=u-m_{H}^{2} .
$$

We have four contributing subprocesses, $g g \rightarrow H g, q g \rightarrow H q, g q \rightarrow H q, q \bar{q} \rightarrow H g$.

The subprocess $\boldsymbol{g} \boldsymbol{g} \rightarrow \boldsymbol{H} \boldsymbol{g}$. The helicity summed (unaveraged) amplitude squared is given by

$$
\left|\mathcal{M}_{g g \rightarrow H g}\right|^{2}=\frac{N_{c}\left(N_{c}^{2}-1\right)}{64 \pi} \alpha_{s}^{3} \sum_{\lambda_{1}, \lambda_{2}, \lambda_{3}= \pm}\left|\sum_{f} M_{g g \rightarrow H g}^{\lambda_{1} \lambda_{2} \lambda_{3}}(s, t, u)\right|^{2},
$$

where the sum is over different quark flavours $f=t, b$ in the loop and $\lambda_{i}$ is the helicity of gluon $p_{i}$.

For a quark of mass $m$ in the loop, coupled to the Higgs with a Yukawa coupling $y=g_{w} m /\left(2 M_{W}\right)$ we have:

$$
\begin{aligned}
\frac{M_{g g \rightarrow H g}^{+++}(s, t, u)}{y m \Delta}= & -64\left(\frac{1}{u t}+\frac{1}{t t_{1}}+\frac{1}{u u_{1}}\right)-\frac{64}{s}\left(\frac{2 s+t}{u_{1}^{2}} B_{1}(u)+\frac{2 s+u}{t_{1}^{2}} B_{1}(t)\right) \\
& -\frac{16\left(s-4 m^{2}\right)}{s t u}\left[s_{1} C_{1}(s)+(u-s) C_{1}(t)+(t-s) C_{1}(u)\right] \\
& -128 m^{2}\left(\frac{1}{t t_{1}} C_{1}(t)+\frac{1}{u u_{1}} C_{1}(u)\right)+\frac{64 m^{2}}{s} D(u, t) \\
& +\frac{8\left(s-4 m^{2}\right)}{s t u}[s t D(s, t)+u s D(u, s)-u t D(u, t)]-\frac{32}{s^{2}} E(u, t),
\end{aligned}
$$


and

$$
\begin{aligned}
\frac{M_{g g \rightarrow H g}^{++-}(s, t, u)}{y m \Delta}= & \frac{64 m_{H}^{2}}{s t u}+\frac{16\left(m_{H}^{2}-4 m^{2}\right)}{s t u}\left[s_{1} C_{1}(s)+t_{1} C_{1}(t)+u_{1} C_{1}(u)\right] \\
& -\frac{8\left(m_{H}^{2}-4 m^{2}\right)}{s t u}[s t D(s, t)+u s D(u, s)+u t D(u, t)]
\end{aligned}
$$

where $\Delta=\sqrt{(s t u) / 8} \cdot B_{1}(s)$ is then defined as:

$$
B_{1}(s)=B(s)-B\left(m_{H}^{2}\right),
$$

where $B$ is the scalar bubble integral

$$
\begin{aligned}
B\left(q^{2}\right) & =\int \frac{d^{4} \ell}{i \pi^{2}} \frac{1}{\left[\ell^{2}-m^{2}\right]\left[(\ell+q)^{2}-m^{2}\right]} \\
& =-\int_{0}^{1} d x \ln \left[m^{2}-i \epsilon-q^{2} x(1-x)\right] .
\end{aligned}
$$

$C$ is the scalar triangle integral

$$
\begin{aligned}
C\left(q^{2}\right) & =\int \frac{d^{4} \ell}{i \pi^{2}} \frac{1}{\left[\ell^{2}-m^{2}\right]\left[\left(\ell+p_{1}\right)^{2}-m^{2}\right]\left[\left(\ell+p_{1}+p_{2}\right)^{2}-m^{2}\right]} \\
& =\int_{0}^{1} \frac{d x}{q^{2} x} \ln \left[1-i \epsilon-\frac{q^{2}}{m^{2}} x(1-x)\right], \quad q^{2}=\left(p_{1}+p_{2}\right)^{2},
\end{aligned}
$$

$C_{1}, D$ and $E$ are other scalar integrals. $C_{1}(s)$ is defined as:

$$
s_{1} C_{1}(s)=s C(s)-m_{H}^{2} C\left(m_{H}^{2}\right),
$$

and an analogous definition holds for $C_{1}(t)$ and $C_{1}(u)$.

$D(s, t)$ is the box integral

$$
\begin{aligned}
D(s, t)= & \int \frac{d^{4} \ell}{i \pi^{2}} \frac{1}{\left[\ell^{2}-m^{2}\right]\left[\left(\ell+p_{1}\right)^{2}-m^{2}\right]\left[\left(\ell+p_{1}+p_{2}\right)^{2}-m^{2}\right]\left[\left(\ell-p_{H}\right)^{2}-m^{2}\right]} \\
= & \frac{1}{s t} \int_{0}^{1} \frac{1}{x(1-x)+m^{2} u /(t s)}\left\{-\ln \left[1-i \epsilon-\frac{m_{H}^{2}}{m^{2}} x(1-x)\right]\right. \\
& \left.\quad+\ln \left[1-i \epsilon-\frac{s}{m^{2}} x(1-x)\right]+\ln \left[1-i \epsilon-\frac{t}{m^{2}} x(1-x)\right]\right\},
\end{aligned}
$$

and analogous definitions hold for $D(u, s)$ and $D(t, u)$.

$E$ is the following linear combination of $C$ and $D$ integrals

$$
E(u, t)=u C(u)+t C(t)+u_{1} C_{1}(u)+t_{1} C_{1}(t)-u t D(u, t) .
$$

The other amplitudes are obtained from these by exchanging the invariants, namely

$$
\begin{aligned}
& M_{g g \rightarrow H g}^{-+-}(s, t, u)=M_{g g \rightarrow H g}^{+++}(t, s, u), \\
& M_{g g \rightarrow H g}^{-++}(s, t, u)=M_{g g \rightarrow H g}^{+++}(u, t, s),
\end{aligned}
$$

and the last four helicity amplitudes can be obtained using parity conservation

$$
M_{g g \rightarrow H g}^{\lambda_{1} \lambda_{2} \lambda_{3}}(s, t, u)=-M_{g g \rightarrow H g}^{-\lambda_{1}-\lambda_{2}-\lambda_{3}}(s, t, u) .
$$


Amplitudes for $\boldsymbol{q} \overline{\boldsymbol{q}} \rightarrow \boldsymbol{H g}$. The amplitude squared for the process $q \bar{q} \rightarrow H g$ is given by

$$
\left|M_{q \bar{q} \rightarrow H g}\right|^{2}(s, t, u)=\frac{N_{c}^{2}-1}{2} \frac{\alpha_{s}^{3}}{\pi} \frac{t^{2}+u^{2}}{s_{1}^{2}} \frac{1}{s}|\mathcal{A}(s, t, u)|^{2},
$$

where

$$
\mathcal{A}(s, t, u)=\sum_{f} y m\left(2+\frac{2 s}{s_{1}} B_{1}(s)+\left(4 m_{f}^{2}-t-u\right) C_{1}(s)\right),
$$

where $m$ denotes the (top or bottom) mass in the loop, $y$ the quark coupling to the Higgs, whereas external quarks are treated as massless.

The matrix elements for the crossed processes $q g \rightarrow H g$ and $g \bar{q} \rightarrow H \bar{q}$ can be obtained from $\left|M_{q \bar{q}}\right|^{2}$ as follows

$$
\begin{aligned}
& \left|M_{q g \rightarrow H q}\right|^{2}(s, t, u)=-\left|M_{q \bar{q} \rightarrow H g}\right|^{2}(u, t, s), \\
& \left|M_{g \bar{q} \rightarrow H \bar{q}}\right|^{2}(s, t, u)=-\left|M_{q \bar{q} \rightarrow H g}\right|^{2}(t, s, u) .
\end{aligned}
$$

\section{B Matching schemes}

In this section we report the expressions for the three matching schemes at NNLL+NNLO defined in ref. [6] and used in this paper. For the purpose of this paper we define

$$
\tilde{\Sigma}_{\mathrm{NNLL}}^{(2)}\left(p_{\mathrm{t}, \text { veto }}\right)=\left.\Sigma_{\mathrm{NNLL}}^{(2)}\left(p_{\mathrm{t}, \text { veto }}\right)\right|_{\text {large }-m_{t}}\left|F_{0}\left(\tau_{t}\right)\right|^{2},
$$

where $\left.\Sigma_{\text {NNLL }}^{(2)}\left(p_{\mathrm{t}, \text { veto }}\right)\right|_{\text {large }-m_{t}}$ is obtained from the second-order expansion of the large- $m_{t}$ resummation formula of ref. [6]. The vetoed cross-section, in the first of the three matching schemes, reads

$$
\begin{aligned}
& \Sigma_{\text {matched }}^{(\mathrm{a})}\left(p_{\mathrm{t}, \text { veto }}\right)=\frac{1}{\sigma_{0}} \frac{\Sigma_{\mathrm{NNLL}}\left(p_{\mathrm{t}, \mathrm{veto}}\right)}{1+\mathcal{L}^{(1)}(\tilde{L}) / \mathcal{L}^{(0)}(\tilde{L})}\left[\sigma_{0}\left(1+\frac{\mathcal{L}^{(1)}(\tilde{L})}{\mathcal{L}^{(0)}(\tilde{L})}\right)+\Sigma^{(1)}\left(p_{\mathrm{t}, \text { veto }}\right)-\Sigma_{\mathrm{NNLL}}^{(1)}\left(p_{\mathrm{t}, \text { veto }}\right)\right. \\
& \left.+\Sigma^{(2)}\left(p_{\mathrm{t}, \text { veto }}\right)-\tilde{\Sigma}_{\mathrm{NNLL}}^{(2)}\left(p_{\mathrm{t}, \text { veto }}\right)+\left(\frac{\mathcal{L}^{(1)}(0)}{\mathcal{L}^{(0)}(0)}-\frac{\Sigma_{\mathrm{NNLL}}^{(1)}\left(p_{\mathrm{t}, \text { veto }}\right)}{\sigma_{0}}\right)\left(\Sigma^{(1)}\left(p_{\mathrm{t}, \text { veto }}\right)-\Sigma_{\mathrm{NNLL}}^{(1)}\left(p_{\mathrm{t}, \text { veto }}\right)\right)\right]
\end{aligned}
$$

where

$$
\tilde{L}=\frac{1}{p} \ln \left[1+\left(\frac{Q}{p_{\text {t,veto }}}\right)^{p}\right] .
$$

For our numerical results we choose $p=5[3]$.

The luminosity factors $\mathcal{L}^{(0)}(\tilde{L})$ and $\mathcal{L}^{(1)}(\tilde{L})$ are defined as

$$
\begin{aligned}
\mathcal{L}^{(0)}(\tilde{L})= & \sum_{i, j} \int d x_{1} d x_{2} \delta\left(x_{1} x_{2} s-M^{2}\right) f_{i}\left(x_{1}, e^{-\tilde{L}} \mu_{F}\right) f_{j}\left(x_{2}, e^{-\tilde{L}} \mu_{F}\right), \\
\mathcal{L}^{(1)}(\tilde{L})= & \frac{\alpha_{s}}{2 \pi} \sum_{i, j} \int d x_{1} d x_{2} \delta\left(x_{1} x_{2} s-M^{2}\right)\left[f_{i}\left(x_{1}, e^{-\tilde{L}} \mu_{F}\right) f_{j}\left(x_{2}, e^{-\tilde{L}} \mu_{F}\right) \mathcal{H}^{(1)}\right. \\
& \left.+\frac{1}{1-2 \alpha_{s} \beta_{0} \tilde{L}} \sum_{k}\left(\int_{x_{1}}^{1} \frac{d z}{z} C_{k i}^{(1)}(z) f_{i}\left(\frac{x_{1}}{z}, e^{-\tilde{L}} \mu_{F}\right) f_{j}\left(x_{2}, e^{-\tilde{L}} \mu_{F}\right)+\left\{\left(x_{1}, i\right) \leftrightarrow\left(x_{2}, j\right)\right\}\right)\right] .
\end{aligned}
$$


The second scheme can be derived from the previous one by replacing $\Sigma^{(2)}\left(p_{\mathrm{t}, \mathrm{veto}}\right)$ with $\bar{\Sigma}^{(2)}\left(p_{\text {t,veto }}\right)$. For the vetoed cross section we get

$$
\begin{aligned}
& \Sigma_{\text {matched }}^{(\mathrm{b})}\left(p_{\mathrm{t}, \text { veto }}\right)=\frac{1}{\sigma_{0}} \frac{\Sigma_{\mathrm{NNLL}}\left(p_{\mathrm{t}, \text { veto }}\right)}{1+\mathcal{L}^{(1)}(\tilde{L}) / \mathcal{L}^{(0)}(\tilde{L})}\left[\sigma_{0}\left(1+\frac{\mathcal{L}^{(1)}(\tilde{L})}{\mathcal{L}^{(0)}(\tilde{L})}\right)+\Sigma^{(1)}\left(p_{\mathrm{t}, \text { veto }}\right)-\Sigma_{\mathrm{NNLL}}^{(1)}\left(p_{\mathrm{t}, \text { veto }}\right)\right. \\
& \left.+\bar{\Sigma}^{(2)}\left(p_{\mathrm{t}, \text { veto }}\right)-\tilde{\Sigma}_{\mathrm{NNLL}}^{(2)}\left(p_{\mathrm{t}, \text { veto }}\right)+\left(\frac{\mathcal{L}^{(1)}(0)}{\mathcal{L}^{(0)}(0)}-\frac{\Sigma_{\mathrm{NNLL}}^{(1)}\left(p_{\mathrm{t}, \text { veto }}\right)}{\sigma_{0}}\right)\left(\Sigma^{(1)}\left(p_{\mathrm{t}, \text { veto }}\right)-\Sigma_{\mathrm{NNLL}}^{(1)}\left(p_{\mathrm{t}, \text { veto }}\right)\right)\right] .
\end{aligned}
$$

Finally, the third matching scheme is directly formulated for the efficiency resulting in

$$
\begin{aligned}
\epsilon_{\text {matched }}^{(c)}\left(p_{t, \text { veto }}\right)=\frac{1}{\sigma_{0}^{2}} \frac{\Sigma_{\text {NNLL }}\left(p_{\mathrm{t}, \text { veto }}\right)}{1+\mathcal{L}^{(1)}(\tilde{L}) / \mathcal{L}^{(0)}(\tilde{L})}\left[\sigma_{0}\left(1+\frac{\mathcal{L}^{(1)}(\tilde{L})}{\mathcal{L}^{(0)}(\tilde{L})}\right)+\bar{\Sigma}^{(1)}\left(p_{\mathrm{t}, \text { veto }}\right)-\Sigma_{\mathrm{NNLL}}^{(1)}\left(p_{\mathrm{t}, \text { veto }}\right)\right. \\
+\bar{\Sigma}^{(2)}\left(p_{\mathrm{t}, \text { veto }}\right)-\frac{\sigma_{1}}{\sigma_{0}} \bar{\Sigma}^{(1)}\left(p_{\mathrm{t}, \text { veto }}\right)-\tilde{\Sigma}_{\mathrm{NNLL}}^{(2)}\left(p_{\mathrm{t}, \text { veto }}\right) \\
\left.+\left(\frac{\mathcal{L}^{(1)}(0)}{\mathcal{L}^{(0)}(0)}-\frac{\Sigma_{\mathrm{NNLL}}^{(1)}\left(p_{\mathrm{t}, \text { veto }}\right)}{\sigma_{0}}\right)\left(\bar{\Sigma}^{(1)}\left(p_{\mathrm{t}, \text { veto }}\right)-\Sigma_{\mathrm{NNLL}}^{(1)}\left(p_{\mathrm{t}, \text { veto }}\right)\right)\right] .
\end{aligned}
$$

The $\log \mathrm{R}-(\mathrm{a})$ scheme discussed in the text to estimate the size of subleading mass effects reads

$$
\begin{aligned}
\Sigma_{\text {matched }}^{\operatorname{logR}-(\mathrm{a})}\left(p_{\mathrm{t}, \text { veto }}\right)= & \frac{1}{\sigma_{0}} \frac{\Sigma_{\mathrm{NNLL}}\left(p_{\mathrm{t}, \text { veto }}\right)}{1+\mathcal{L}^{(1)}(\tilde{L}) / \mathcal{L}^{(0)}(\tilde{L})}\left[\sigma_{0}\left(1+\frac{\mathcal{L}^{(1)}(\tilde{L})}{\mathcal{L}^{(0)}(\tilde{L})}\right)+\Sigma^{(2)}\left(p_{\mathrm{t}, \text { veto }}\right)-\tilde{\Sigma}_{\mathrm{NNLL}}^{(2)}\left(p_{\mathrm{t}, \text { veto }}\right)\right. \\
& \left.-\frac{\Sigma_{\mathrm{NNLL}}^{(1)}\left(p_{\mathrm{t}, \text { veto }}\right)}{\sigma_{0}}\left(\Sigma^{(1)}\left(p_{\mathrm{t}, \text { veto }}\right)-\Sigma_{\mathrm{NNLL}}^{(1)}\left(p_{\mathrm{t}, \text { veto }}\right)\right)\right] \\
& \exp \left\{\frac{\Sigma^{(1)}\left(p_{\mathrm{t}, \text { veto }}\right)-\Sigma_{\mathrm{NNLL}}^{(1)}\left(p_{\mathrm{t}, \text { veto }}\right)}{\sigma_{0}}-\frac{1}{2}\left(\frac{\Sigma^{(1)}\left(p_{\mathrm{t}, \text { veto }}\right)-\Sigma_{\mathrm{NNLL}}^{(1)}\left(p_{\mathrm{t}, \text { veto }}\right)}{\sigma_{0}}\right)^{2}\right\} .
\end{aligned}
$$

Notice that the (c) scheme is directly expressed in terms of the jet veto efficiency, while for the schemes (a), (b), $\operatorname{logR}$-(a) and mod-(a) eq. (C.1) the corresponding efficiencies are defined as

$$
\epsilon_{\text {matched }}\left(p_{\mathrm{t}, \mathrm{veto}}\right)=\frac{\Sigma_{\text {matched }}\left(p_{\mathrm{t}, \mathrm{veto}}\right)}{\Sigma_{\text {matched }}\left(p_{\mathrm{t}, \mathrm{veto}}^{\max }\right)},
$$

where $p_{\mathrm{t}, \mathrm{veto}}^{\max }$ is maximum kinematically allowed jet transverse momentum.

\section{Mod-(a) matching scheme}

In the matching schemes of ref. [6] used here, for $p_{\mathrm{t} \text {,veto }} \ll m_{H}$ the remainder is multiplied by both a Sudakov form factor and a luminosity prefactor (see eqs. (B.2), (B.6), (B.7)). It is actually not known whether this structure is fulfilled by higher order corrections. One might argue that multiplying the remainder by a Sudakov form factor is appropriate, since the latter represents the probability of having no emissions at scales above $p_{\mathrm{t} \text {,veto }}$. On the other hand, the luminosity prefactor accounts for all collinear splittings which

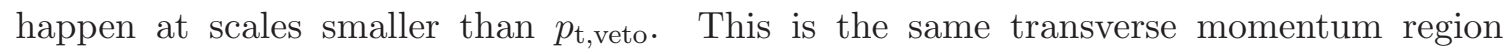
which contributes to the remainder function. Therefore, it might seem more appropriate to devise a new matching scheme where the remainder is not multiplied neither by the Sudakov form factor nor by a luminosity prefactor. 


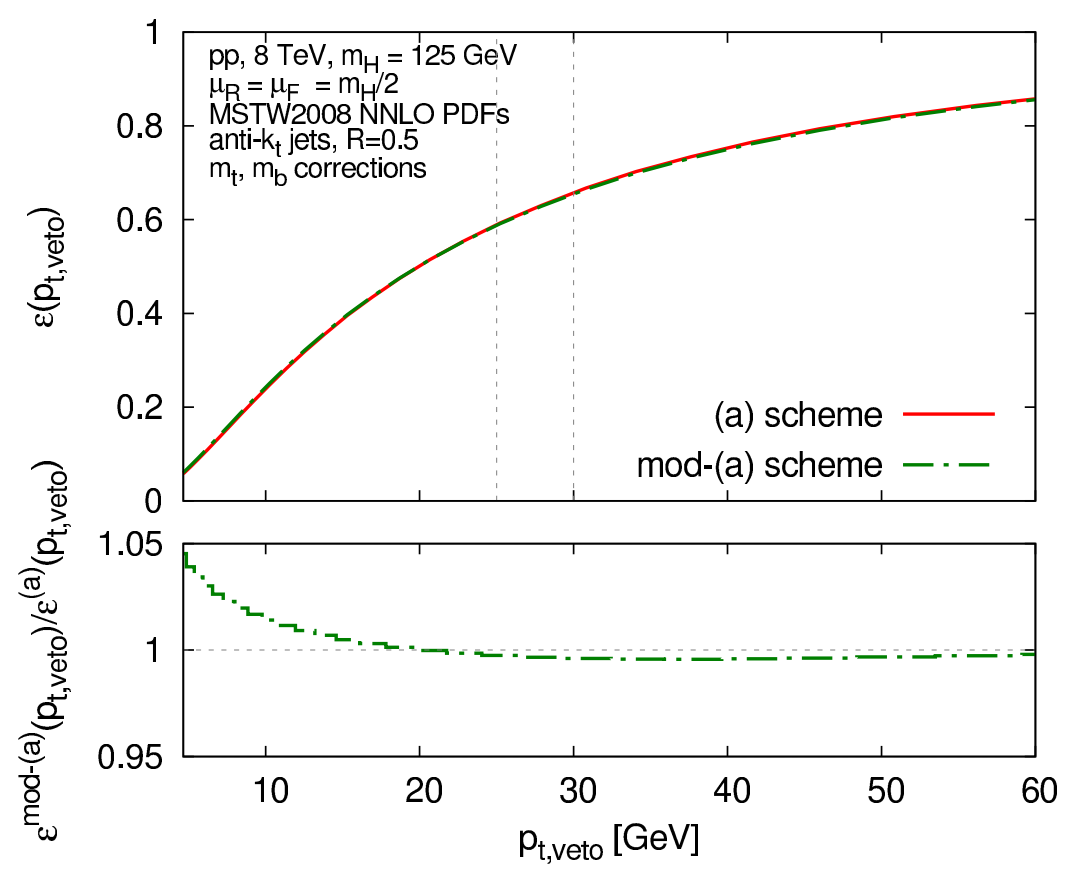

Figure 10. Matched jet veto efficiency obtained with two different matching schemes. The two schemes differ in the treatment of the NLO bottom-mass-dependent remainder as discussed in the text.

The new hybrid scheme, referred to as mod-(a) scheme, is a modification of the (a) scheme of ref. [6], where the $\mathcal{O}\left(\alpha_{s}^{3}\right)$ regular remainder is treated additively. We hence introduce the mod-(a) scheme as

$$
\begin{aligned}
\Sigma_{\text {matched }}^{\text {mod }-(a)}\left(p_{\mathrm{t}, \text { veto }}\right)=\frac{1}{\sigma_{0}} \frac{\Sigma_{\mathrm{NNLL}}\left(p_{\mathrm{t}, \text { veto }}\right)}{1+\mathcal{L}^{(1)}(\tilde{L}) / \mathcal{L}^{(0)}(\tilde{L})}\left[\sigma_{0}\left(1+\frac{\mathcal{L}^{(1)}(\tilde{L})}{\mathcal{L}^{(0)}(\tilde{L})}\right)+\right. & \left.\Sigma^{(2)}\left(p_{\mathrm{t}, \text { veto }}\right)-\tilde{\Sigma}_{\mathrm{NNLL}}^{(2)}\left(p_{\mathrm{t}, \text { veto }}\right)\right] \\
& +\Sigma^{(1)}\left(p_{\mathrm{t}, \text { veto }}\right)-\Sigma_{\mathrm{NNLL}}^{(1)}\left(p_{\mathrm{t}, \text { veto }}\right) .
\end{aligned}
$$

This scheme is defined as scheme (a), but the one-loop remainder terms are not multiplied by the luminosity or Sudakov form factors. Instead, the two-loop remainder is treated in a multiplicative way, as in the original formulation of the (a) scheme. In this way, the matched cross-section contains all logarithmically enhanced contributions in $\Sigma^{(J)}\left(p_{\text {t,veto }}\right)$ up to order $\alpha_{s}^{n} L^{2 n-3}$. We remind however that the fixed-order results that we use do not contain the correct $\mathcal{O}\left(\alpha_{s}^{4}\right)$ constant term with full dependence on the heavy-quark masses. Therefore the resulting two-loop constant terms is rescaled according to the correction factor $\left|F_{0}\left(\tau_{t}\right)\right|^{2}$ where $F_{0}\left(\tau_{t}\right)$ is defined in (4.3).

Figure 10 shows the comparison between the mod-(a) scheme and the (a) scheme for the matched jet veto efficiency. The difference between the two schemes gives us an estimate of whether it is numerically relevant to multiply the remainder by the Sudakov and luminosity factors, or not. We see that the two results differ at the few-percent level for very small $p_{\text {t,veto }} \lesssim 10 \mathrm{GeV}$, but only at per-mille level in the region of interest $p_{\mathrm{t} \text {,veto }} \sim 25-30 \mathrm{GeV}$. 


\section{Comparison to the Higgs transverse momentum}

It is interesting to investigate whether, in our framework, mass-effects affect the Higgs transverse momentum distribution in a similar way to the jet-veto distribution. We have therefore extended the JetVHeto code to produce resummed and matched results for the distribution in the Higgs transverse momentum $p_{t, H}$, including mass effects. For this purpose, we have used eq. (43) of ref. [6], with the same one-loop coefficient constants as for jet-veto distribution $\left(\mathcal{H}^{(1)}\right.$ and $C_{k i}^{(1)}(z)$ in eq. (4.1)).

We first note that, already at NLL, the multiple-emission function for $p_{t, H}, \mathcal{F}\left(R^{\prime}\right)=$ $e^{-\gamma_{E} R^{\prime}} \Gamma\left(1-R^{\prime} / 2\right) / \Gamma\left(1+R^{\prime} / 2\right)$, has a divergence at $R^{\prime}=2$, which corresponds to $p_{t, H} \sim$ $5 \mathrm{GeV}$ for a resummation scale $Q=m_{H} / 2$. Decreasing the value of $Q$ pushes the divergence to even lower values of $p_{t, H}$. The origin of this divergence is well understood [47] and is explained in detail in the case of the oblateness in ref. [48]. It is related to the fact that multiple emissions contribute vectorially to $p_{t, H}$, therefore a small value of $p_{t, H}$ can also arise in configurations involving emissions with large $p_{t}$ that cancel against each other. When this mechanism dominates over the standard Sudakov suppression (events for which the low $p_{t, H}$ is due to low $p_{t}$ emissions), one can not use the normal logarithmic hierarchy to establish which logarithmic terms are dominant. The divergence in $\mathcal{F}\left(R^{\prime}\right)$ is related to using a formula that assumes this hierarchy, obtained by neglecting subleading effects.

Since our prediction diverges at $p_{t, H} \sim 5 \mathrm{GeV}$, we consider it unreliable for $p_{t, H} \lesssim 15 \mathrm{GeV}$. The excluded region is denoted by a gray band in the plots below.

In the following, we wish to investigate the origin of the large discrepancy between matched and fixed-order distributions observed by the authors of ref. [30], when using a resummation scale of order $m_{H}$. We then consider matched distributions obtained with $\mu_{R}=\mu_{F}=m_{H}$ and resummation scales $Q_{1}=Q_{2}=m_{H} / 2$ (where $Q_{1}$ and $Q_{2}$ are the resummation scales corresponding to the top and bottom-induced contributions, respectively). More specifically, in figures 11 (12) we show the NLL+NLO and NLO (NLL+NNLO, NNLL+NNLO and NNLO) differential distributions for $p_{t, H}$ (upper plots) and $p_{\mathrm{t} \text {,jet }}$ (lower plots) in the large- $m_{t}$ limit (left-hand plots), and including finite $m_{t}, m_{b}$ effects (central plots). Since the bottom-induced contribution is only a small fraction of the total, it is useful to also plot this separately (right-hand plots). We observe that all of the NLL+NLO matched results differ considerably from the NLO in the intermediate $p_{t}$ region $\left(20 \mathrm{GeV} \lesssim p_{t} \lesssim 50 \mathrm{GeV}\right)$. This happens regardless of the observable, $p_{t, H}$ or $p_{\text {t,jet, }}$, and of whether one includes mass-effects, or not. We therefore conclude that this discrepancy cannot be ascribed to a large non-factorizing correction, which is present only in the bottom-induced contributions. The difference between the matched and fixed-order results is due to missing higher order terms.

In the large- $m_{t}$ limit (and in all standard cases), when a matching is performed at the NLL+NLO (NNLL+NNLO) level, this difference is of relative order $\mathcal{O}\left(\alpha_{s}^{2}\right)\left(\mathcal{O}\left(\alpha_{s}^{3}\right)\right)$. Therefore we do observe a better convergence with a NNLL+NNLO matching, as one would expect (see left-hand plots of figure 12). Unfortunately, the full NNLO correction $\left(\mathcal{O}\left(\alpha_{s}^{3}\right)\right)$ including exact mass effects is so far unknown. Therefore we use the NNLO result in the large $m_{t}$-limit, rescaled by the ratio of the Born cross-section including exact 

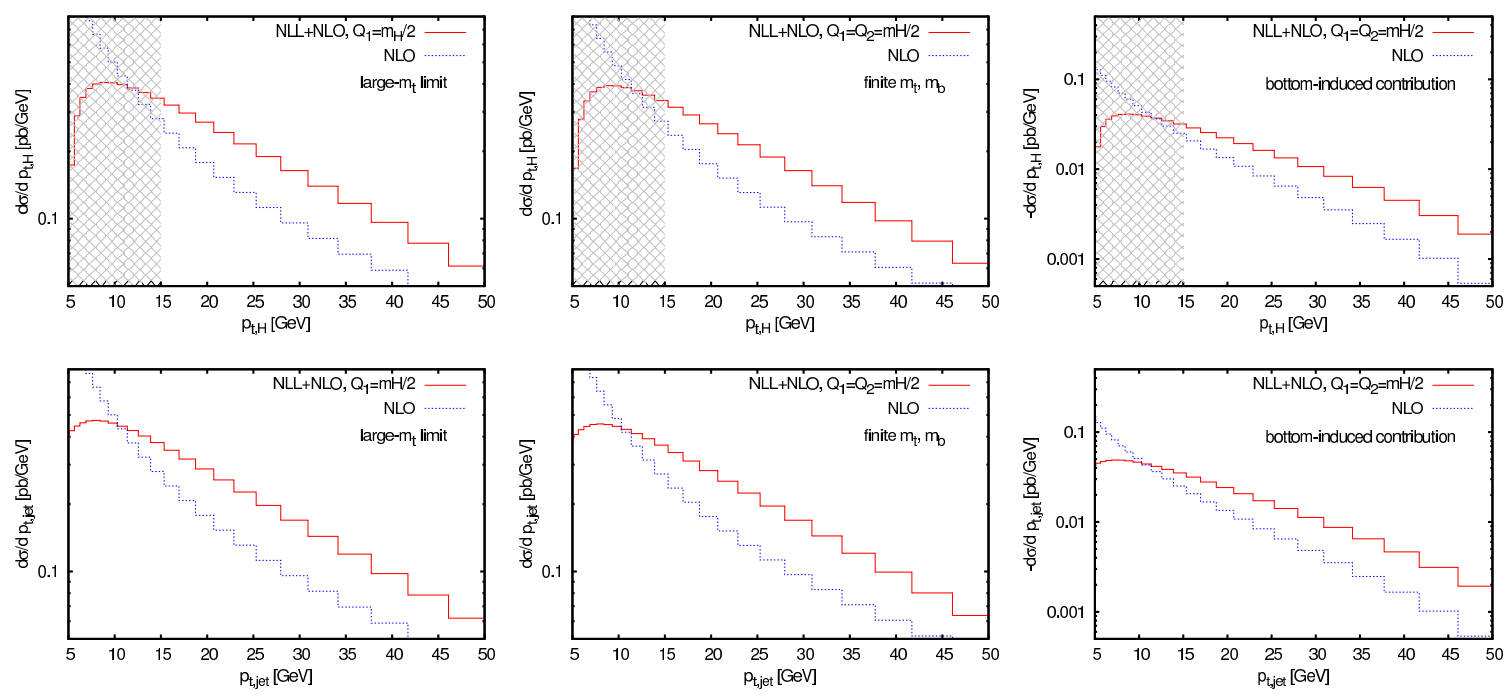

Figure 11. NLL+NLO (red) and NLO (blue) differential distributions for $p_{t, H}$ (upper plots) and $p_{\mathrm{t}, \text { jet }}$ (lower plots) in the large- $m_{t}$ limit (left-hand plots), including finite $m_{t}, m_{b}$ effects (central plots) and bottom-induced contribution only (right-hand plots).
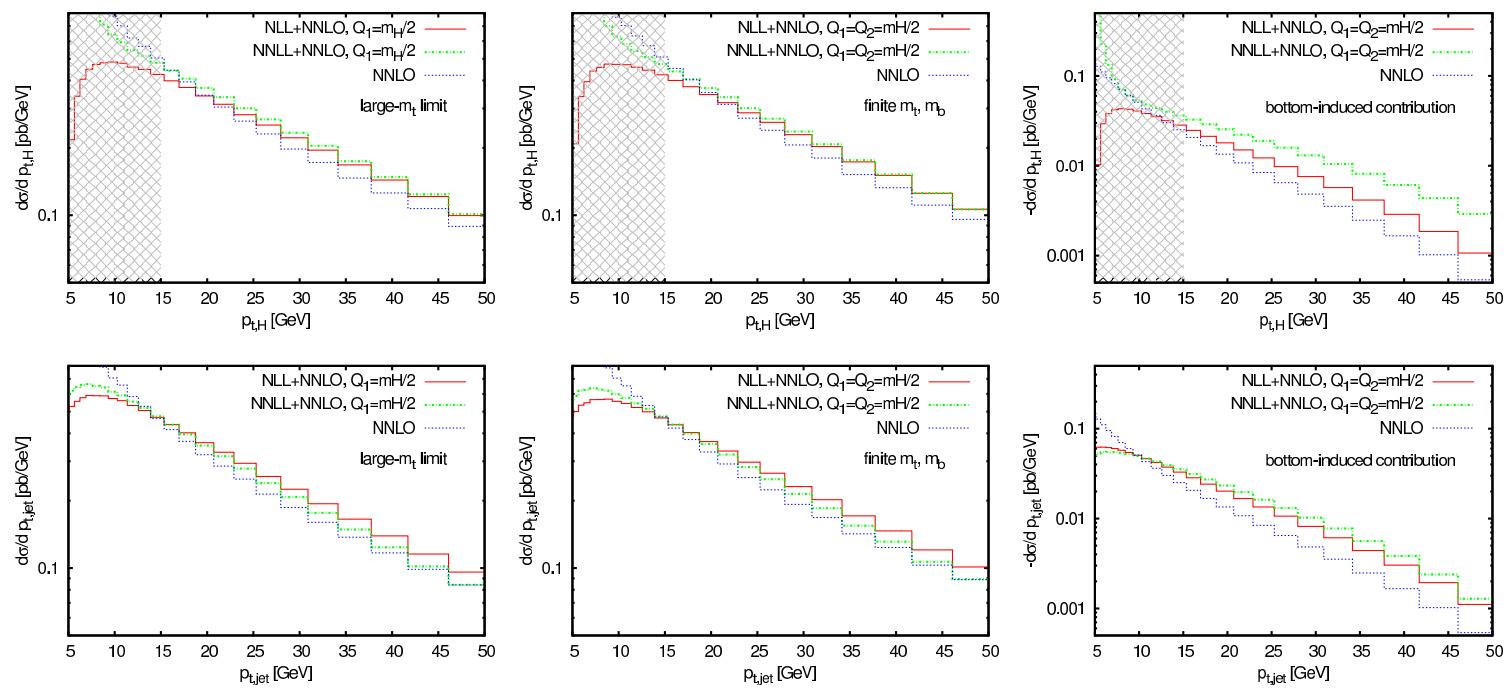

Figure 12. NLL+NNLO (red), NNLL+NNLO (green) and NNLO (blue) differential distributions for $p_{t, H}$ (upper plots) and $p_{\mathrm{t} \text {,jet }}$ (lower plots) in the large- $m_{t}$ limit (left-hand plots), including finite $m_{t}, m_{b}$ effects (central plots) and bottom-induced contribution only (right-hand plots).

top-mass effects to the Born cross-section in the large $m_{t}$ limit. When matching to this NNLO correction, the expansion of our resummation formula at $\mathcal{O}\left(\alpha_{s}^{2}\right)$ appearing in our matching formulae is replaced by the corresponding one in the large $m_{t}$ limit multiplied by the same rescaling factor. This is to guarantee NNLL accuracy in the Sudakov region. However, this implies that even when matching at NNLO+NNLL level, the discrepancy between fixed-order and matched results is formally still $\mathcal{O}\left(\alpha_{s}^{2}\right)$.

In order to investigate whether this is really the source of the observed difference between matched and fixed-order distributions, we can use the actual expansion of our 

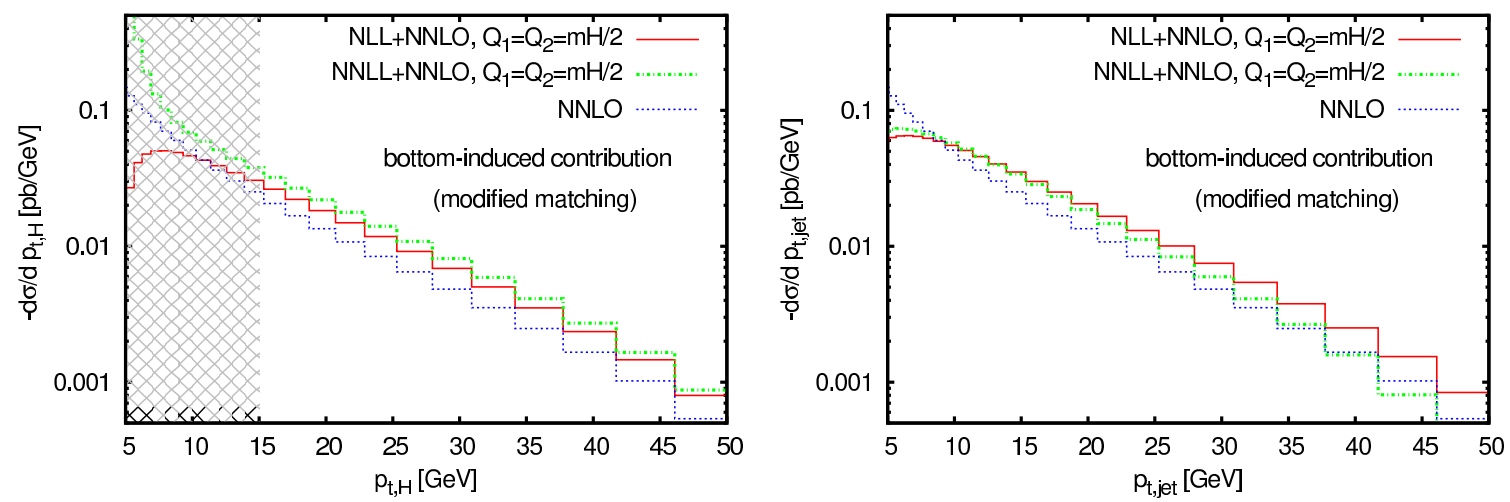

Figure 13. NLL+NNLO (red), NNLL+NNLO (green) and NNLO (blue) differential distributions for the bottom-induced contribution for $p_{t, H}$ (left) and $p_{\mathrm{t} \text {,jet }}$ (right) using the actual expansion of the resummation formula when matching to NNLO (see text for more details).

resummation (which however spoils the logarithmic accuracy in the low $p_{t}$ region). This ensures that, in the medium-large $p_{t}$ region, the correct fixed-order behavior is reproduced, and the left-over is of $\mathcal{O}\left(\alpha_{s}^{3}\right)$. The outcome of this exercise is shown in figure 13 for the bottom-induced contribution. As expected, the discrepancy between matched and fixed-order is now similar to the one observed in the large $m_{t}$ limit.

Open Access. This article is distributed under the terms of the Creative Commons Attribution License (CC-BY 4.0), which permits any use, distribution and reproduction in any medium, provided the original author(s) and source are credited.

\section{References}

[1] ATLAS collaboration, Observation of a new particle in the search for the Standard Model Higgs boson with the ATLAS detector at the LHC, Phys. Lett. B 716 (2012) 1 [arXiv:1207.7214] [INSPIRE].

[2] CMS collaboration, Observation of a new boson at a mass of $125 \mathrm{GeV}$ with the CMS experiment at the LHC, Phys. Lett. B $\mathbf{7 1 6}$ (2012) 30 [arXiv:1207.7235] [INSPIRE].

[3] A. Banfi, G.P. Salam and G. Zanderighi, $N L L+N N L O$ predictions for jet-veto efficiencies in Higgs-boson and Drell-Yan production, JHEP 06 (2012) 159 [arXiv:1203.5773] [INSPIRE].

[4] A. Banfi, G.P. Salam and G. Zanderighi, Principles of general final-state resummation and automated implementation, JHEP 03 (2005) 073 [hep-ph/0407286] [INSPIRE].

[5] T. Becher and M. Neubert, Factorization and NNLL resummation for Higgs production with a jet veto, JHEP 07 (2012) 108 [arXiv:1205.3806] [INSPIRE].

[6] A. Banfi, P.F. Monni, G.P. Salam and G. Zanderighi, Higgs and Z-boson production with a jet veto, Phys. Rev. Lett. 109 (2012) 202001 [arXiv:1206.4998] [INSPIRE].

[7] I.W. Stewart, F.J. Tackmann, J.R. Walsh and S. Zuberi, Jet $p_{T}$ resummation in Higgs production at $N N L L^{\prime}+N N L O$, arXiv:1307.1808 [INSPIRE].

[8] T. Becher, M. Neubert and L. Rothen, Factorization and $N^{3} L L_{p}+N N L O$ predictions for the Higgs cross section with a jet veto, JHEP 10 (2013) 125 [arXiv:1307.0025] [INSPIRE]. 
[9] M. Cacciari, G.P. Salam and G. Soyez, The anti- $k_{T}$ jet clustering algorithm, JHEP 04 (2008) 063 [arXiv:0802.1189] [INSPIRE].

[10] M. Spira, A. Djouadi, D. Graudenz and P. Zerwas, Higgs boson production at the LHC, Nucl. Phys. B 453 (1995) 17 [hep-ph/9504378] [INSPIRE].

[11] M. Spira, QCD effects in Higgs physics, Fortsch. Phys. 46 (1998) 203 [hep-ph/9705337] [INSPIRE].

[12] R. Harlander and P. Kant, Higgs production and decay: analytic results at next-to-leading order QCD, JHEP 12 (2005) 015 [hep-ph/0509189] [INSPIRE].

[13] C. Anastasiou, S. Beerli, S. Bucherer, A. Daleo and Z. Kunszt, Two-loop amplitudes and master integrals for the production of a Higgs boson via a massive quark and a scalar-quark loop, JHEP 01 (2007) 082 [hep-ph/0611236] [INSPIRE].

[14] U. Aglietti, R. Bonciani, G. Degrassi and A. Vicini, Analytic results for virtual QCD corrections to Higgs production and decay, JHEP 01 (2007) 021 [hep-ph/0611266] [INSPIRE].

[15] R. Bonciani, G. Degrassi and A. Vicini, Scalar particle contribution to Higgs production via gluon fusion at NLO, JHEP 11 (2007) 095 [arXiv: 0709.4227] [INSPIRE].

[16] R.V. Harlander, T. Neumann, K.J. Ozeren and M. Wiesemann, Top-mass effects in differential Higgs production through gluon fusion at order $\alpha_{s}^{4}$, JHEP 08 (2012) 139 [arXiv: 1206.0157] [INSPIRE].

[17] MCFM - Monte Carlo for FeMtobarn processes webpage, http://mcfm.fnal.gov/.

[18] C. Anastasiou, S. Bucherer and Z. Kunszt, HPro: a NLO Monte-Carlo for Higgs production via gluon fusion with finite heavy quark masses, JHEP 10 (2009) 068 [arXiv:0907.2362] [INSPIRE].

[19] R.V. Harlander, S. Liebler and H. Mantler, SusHi: a program for the calculation of Higgs production in gluon fusion and bottom-quark annihilation in the Standard Model and the MSSM, Comput. Phys. Commun. 184 (2013) 1605 [arXiv:1212.3249] [INSPIRE].

[20] R.V. Harlander and W.B. Kilgore, Higgs boson production in bottom quark fusion at next-to-next-to leading order, Phys. Rev. D 68 (2003) 013001 [hep-ph/0304035] [INSPIRE].

[21] R.V. Harlander, K.J. Ozeren and M. Wiesemann, Higgs plus jet production in bottom quark annihilation at next-to-leading order, Phys. Lett. B 693 (2010) 269 [arXiv:1007.5411] [INSPIRE].

[22] R. Harlander and M. Wiesemann, Jet-veto in bottom-quark induced Higgs production at next-to-next-to-leading order, JHEP 04 (2012) 066 [arXiv:1111.2182] [INSPIRE].

[23] S. Bühler, F. Herzog, A. Lazopoulos and R. Müller, The fully differential hadronic production of a Higgs boson via bottom quark fusion at NNLO, JHEP 07 (2012) 115 [arXiv:1204.4415] [INSPIRE].

[24] G. Corcella et al., HERWIG 6: an event generator for hadron emission reactions with interfering gluons (including supersymmetric processes), JHEP 01 (2001) 010 [hep-ph/0011363] [INSPIRE].

[25] G. Corcella et al., HERWIG 6.5 release note, hep-ph/0210213 [INSPIRE].

[26] T. Sjöstrand, S. Mrenna and P.Z. Skands, PYTHIA 6.4 physics and manual, JHEP 05 (2006) 026 [hep-ph/0603175] [INSPIRE]. 
[27] E. Bagnaschi, G. Degrassi, P. Slavich and A. Vicini, Higgs production via gluon fusion in the POWHEG approach in the SM and in the MSSM, JHEP 02 (2012) 088 [arXiv:1111.2854] [INSPIRE].

[28] The MC@NLO package version 4.08 webpage, http://www.hep.phy.cam.ac.uk/theory/webber/MCatNLO/.

[29] H. Mantler and M. Wiesemann, Top- and bottom-mass effects in hadronic Higgs production at small transverse momenta through LO+NLL, Eur. Phys. J. C 73 (2013) 2467 [arXiv: 1210.8263] [INSPIRE].

[30] M. Grazzini and H. Sargsyan, Heavy-quark mass effects in Higgs boson production at the LHC, JHEP 09 (2013) 129 [arXiv:1306.4581] [INSPIRE].

[31] R.K. Ellis, Z. Kunszt, K. Melnikov and G. Zanderighi, One-loop calculations in quantum field theory: from Feynman diagrams to unitarity cuts, Phys. Rept. 518 (2012) 141 [arXiv:1105.4319] [INSPIRE].

[32] R.K. Ellis, I. Hinchliffe, M. Soldate and J. van der Bij, Higgs decay to $\tau^{+} \tau^{-}$: a possible signature of intermediate mass Higgs bosons at the SSC, Nucl. Phys. B 297 (1988) 221 [INSPIRE].

[33] U. Baur and E.N. Glover, Higgs boson production at large transverse momentum in hadronic collisions, Nucl. Phys. B 339 (1990) 38 [INSPIRE].

[34] S. Catani, L. Trentadue, G. Turnock and B. Webber, Resummation of large logarithms in $e^{+} e^{-}$event shape distributions, Nucl. Phys. B 407 (1993) 3 [inSPIRE].

[35] A. Martin, W. Stirling, R. Thorne and G. Watt, Parton distributions for the LHC, Eur. Phys. J. C 63 (2009) 189 [arXiv:0901.0002] [InSPIRE].

[36] M. Cacciari and G.P. Salam, Dispelling the $N^{3}$ myth for the $k_{T}$ jet-finder, Phys. Lett. B 641 (2006) 57 [hep-ph/0512210] [INSPIRE].

[37] M. Cacciari, G.P. Salam and G. Soyez, FastJet user manual, Eur. Phys. J. C 72 (2012) 1896 [arXiv:1111.6097] [INSPIRE].

[38] S. Catani and M. Grazzini, An NNLO subtraction formalism in hadron collisions and its application to Higgs boson production at the LHC, Phys. Rev. Lett. 98 (2007) 222002 [hep-ph/0703012] [INSPIRE].

[39] LhC Higgs Cross Section Working Group collaboration, S. Heinemeyer et al., Handbook of LHC Higgs cross sections: 3. Higgs properties, arXiv: 1307.1347 [INSPIRE].

[40] P. Nason, A new method for combining NLO QCD with shower Monte Carlo algorithms, JHEP 11 (2004) 040 [hep-ph/0409146] [INSPIRE].

[41] S. Alioli, P. Nason, C. Oleari and E. Re, A general framework for implementing NLO calculations in shower Monte Carlo programs: the POWHEG BOX, JHEP 06 (2010) 043 [arXiv: 1002.2581] [INSPIRE].

[42] S. Frixione and B.R. Webber, Matching NLO QCD computations and parton shower simulations, JHEP 06 (2002) 029 [hep-ph/0204244] [INSPIRE].

[43] K. Hamilton, P. Nason and G. Zanderighi, MINLO: multi-scale improved NLO, JHEP 10 (2012) 155 [arXiv:1206.3572] [INSPIRE]. 
[44] K. Hamilton, P. Nason, C. Oleari and G. Zanderighi, Merging $H / W / Z+0$ and 1 jet at $N L O$ with no merging scale: a path to parton shower + NNLO matching, JHEP 05 (2013) 082 [arXiv: 1212.4504] [INSPIRE].

[45] A. Vicini, Quark-mass effects in POWHEG and Hres results, talk given at ggF meeting, http://indico.cern.ch/getFile.py/access?contribId=2\&resId=0\&materialId=slides \&confId=263472, CERN Geneva Switzerland July 232013.

[46] JetVHeto webpage, http://jetvheto.hepforge.org/.

[47] S. Frixione, P. Nason and G. Ridolfi, Problems in the resummation of soft gluon effects in the transverse momentum distributions of massive vector bosons in hadronic collisions, Nucl. Phys. B 542 (1999) 311 [hep-ph/9809367] [InSPIRE].

[48] A. Banfi, G. Salam and G. Zanderighi, Semi-numerical resummation of event shapes, JHEP 01 (2002) 018 [hep-ph/0112156] [INSPIRE]. 